\title{
Increasing and decreasing subsequences and their variants
}

\author{
Richard P. Stanley
}

\begin{abstract}
We survey the theory of increasing and decreasing subsequences of permutations. Enumeration problems in this area are closely related to the RSK algorithm. The asymptotic behavior of the expected value of the length is $(w)$ of the longest increasing subsequence of a permutation $w$ of $1,2, \ldots, n$ was obtained by Vershik-Kerov and (almost) by Logan-Shepp. The entire limiting distribution of is $(w)$ was then determined by Baik, Deift, and Johansson. These techniques can be applied to other classes of permutations, such as involutions, and are related to the distribution of eigenvalues of elements of the classical groups. A number of generalizations and variations of increasing/decreasing subsequences are discussed, including the theory of pattern avoidance, unimodal and alternating subsequences, and crossings and nestings of matchings and set partitions.
\end{abstract}

Mathematics Subject Classification (2000). Primary 05A05, 05A06; Secondary 60C05.

Keywords. Increasing subsequence, decreasing subsequence, Young tableau, RSK algorithm, Tracy-Widom distribution, GUE model, pattern avoidance, unimodal subsequence, alternating subsequence, matching, oscillating tableau.

\section{Introduction}

Let $\mathfrak{S}_{n}$ denote the symmetric group of all permutations of $[n]:=\{1,2, \ldots, n\}$. We write permutations $w \in \mathfrak{S}_{n}$ as words, i.e., $w=a_{1} a_{2} \ldots a_{n}$, where $w(i)=a_{i}$. An increasing subsequence of $w$ is a subsequence $a_{i_{1}} \ldots a_{i_{k}}$ satisfying $a_{i_{1}}<\cdots<a_{i_{k}}$, and similarly for decreasing subsequence. For instance, if $w=5642713$, then 567 is an increasing subsequence and 543 is a decreasing subsequence. Let is $(w)$ (respectively, $\mathrm{ds}(w)$ ) denote the length (number of terms) of the longest increasing (respectively, decreasing) subsequence of $w$. If $w=5642713$ as above, then is $(w)=3$ (corresponding to 567) and $\mathrm{ds}(w)=4$ (corresponding to 5421 or 6421 ). A nice interpretation of increasing subsequences in terms of the one-person card game patience sorting is given by Aldous and Diaconis [4]. Further work on patience sorting was undertaken by Burstein and Lankham [33], [34], [35]. Connections between patience sorting and airplane boarding times were found by Bachmat et al. [12], [13] and between patience sorting and disk scheduling by Bachmat [11].

The subject of increasing and decreasing subsequences began in 1935, and there has been much recent activity. There have been major breakthroughs in understanding

Proceedings of the International Congress of Mathematicians, Madrid, Spain, 2006

(C) 2006 European Mathematical Society 
the distribution of is $(w)$, ds $(w)$, and related statistics on permutations, and many unexpected and deep connections have been obtained with such areas as representation theory and random matrix theory. A number of excellent survey papers have been written on various aspects of these developments, e.g., [4], [40], [60], [108], [115]; the present paper will emphasize the connections with combinatorics.

In Section 2 we give some basic enumerative results related to increasing/decreasing subsequences and show their connection with the RSK algorithm from algebraic combinatorics. The next two sections are devoted to the distribution of is $(w)$ for $w \in \mathfrak{S}_{n}$, a problem first raised by Ulam. In Section 3 we deal with the expectation of is $(w)$ for $w \in \mathfrak{S}_{n}$, culminating in the asymptotic formula of Logan-Shepp and Vershik-Kerov. We turn to the entire limiting distribution of is $(w)$ in Section 4. The main result is the determination of this limiting distribution by Baik, Deift, and Johansson to be a (suitably scaled) Tracy-Widom distribution. The Tracy-Widom distribution originally arose in the theory of random matrices, so the result of Baik et al. opens up unexpected connections between increasing/decreasing subsequences and random matrices.

Much of the theory of increasing/decreasing subsequences of permutations in $\mathfrak{S}_{n}$ carries over to permutations in certain subsets of $\mathfrak{S}_{n}$, such as the set of involutions. This topic is discussed in Section 5. In particular, analogues of the Baik-DeiftJohansson theorem were given by Baik and Rains. In Section 6 we explain how the previous results are related to the distribution of eigenvalues in matrices belonging to the classical groups.

The remaining three sections are concerned with analogues and extensions of increasing/decreasing subsequences of permutations. Section 7 deals with pattern avoidance, where increasing/decreasing subsequence are replaced with other "patterns." In Section 8 we consider unimodal and alternating subsequences of permutations, and in Section 9 we replace permutations with (complete) matchings. For matchings the role of increasing and decreasing subsequences is played by crossings and nestings.

Acknowledgment. I am grateful to Percy Deift, Persi Diaconis, Craig Tracy and Herb Wilf for providing some pertinent references.

\section{Enumeration and the RSK algorithm}

The first result on increasing and decreasing subsequences is a famous theorem of Erdôs and Szekeres [43].

Theorem 1. Let $p, q \geq 1$. If $w \in \mathfrak{S}_{p q+1}$, then either is $(w)>p$ or $\operatorname{ds}(w)>q$.

This result arose in the context of the problem of determining the least integer $f(n)$ so that any $f(n)$ points in general position in the plane contain an $n$-element subset $S$ in convex position (i.e., every element of $S$ is a vertex of the convex hull of $S$ ). A recent 
survey of this problem was given by Morris and Soltan [77]. Seidenberg [92] gave an exceptionally elegant proof of Theorem 1 based on the pigeonhole principle which has been reproduced many times, e.g., Gardner [46, Ch. 11, §7]. For a mathematical game based on Theorem 1, see the papers [2], [55].

Theorem 1 is best possible in that there exists $w \in \mathfrak{S}_{p q}$ with is $(w)=p$ and $\operatorname{ds}(w)=q$. Schensted [89] found a quantitative strengthening of this result based on his rediscovery of an algorithm of Robinson [84] which has subsequently become a central algorithm in algebraic combinatorics. To describe Schensted's result, let $\lambda=\left(\lambda_{1}, \lambda_{2}, \ldots\right)$ be a partition of $n \geq 0$, denoted $\lambda \vdash n$ or $|\lambda|=n$. Thus $\lambda_{1} \geq \lambda_{2} \geq$ $\cdots \geq 0$ and $\sum \lambda_{i}=n$. The (Young) diagram) of a partition $\lambda$ is a left-justified array of squares with $\lambda_{i}$ squares in the $i$ th row. For instance, the Young diagram of $(3,2,2)$ is given by

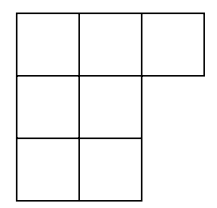

A standard Young tableau (SYT) of shape $\lambda \vdash n$ is obtained by placing the integers $1,2, \ldots, n$ (each appearing once) into the squares of the diagram of $\lambda$ (with one integer in each square) such that every row and column is increasing. For example, an SYT of shape $(3,2,2)$ is given by

$$
\begin{array}{ll}
1 & 3 \\
2 & 5 \\
4 & 7
\end{array}
$$

Let $f^{\lambda}$ denote the number of SYT of shape $\lambda$. The quantity $f^{\lambda}$ has a number of additional combinatorial interpretations [99, Prop. 7.10.3]. It also has a fundamental algebraic interpretation which suggests (via equation (9) below) a close connection between representation theory and increasing/decreasing subsequences. Namely, the (complex) irreducible representations $F^{\lambda}$ of $\mathfrak{S}_{n}$ are indexed in a natural way by partitions $\lambda \vdash n$, and then

$$
f^{\lambda}=\operatorname{dim} F^{\lambda} .
$$

In particular (by elementary representation theory),

$$
\sum_{\lambda \vdash n}\left(f^{\lambda}\right)^{2}=n !
$$

See Section 6 for more on the connections between increasing/decreasing subsequences and representation theory. MacMahon [74, p. 175] was the first to give a formula for $f^{\lambda}$ (in the guise of counting "lattice permutations" rather than SYT), and later Frame, Robinson, and Thrall [45] simplified MacMahon's formula, as follows. Let $u$ be a square of the diagram of $\lambda$, denoted $u \in \lambda$. The hook length $h(u)$ of (or 
at) $u$ is the number of squares directly to the right or directly below $u$, counting $u$ itself once. For instance, if $\lambda=(3,2,2)$ then the hook lengths are given by

\section{1 \\ 32 \\ 21.}

The hook-length formula of Frame, Robinson, and Thrall asserts that if $\lambda \vdash n$, then

$$
f^{\lambda}=\frac{n !}{\prod_{u \in \lambda} h(u)} .
$$

For instance,

$$
f^{(3,2,2)}=\frac{7 !}{5 \cdot 4 \cdot 1 \cdot 3 \cdot 2 \cdot 2 \cdot 1}=21 .
$$

The RSK algorithm gives a bijection between $\mathfrak{S}_{n}$ and pairs $(P, Q)$ of SYT of the same shape $\lambda \vdash n$. This algorithm is named after Gilbert de Beauregard Robinson, who described it in a rather vague form [84, §5] (subsequently analyzed by van Leeuwen [70, §7]), Craige Schensted [89], and Donald Knuth [65]. For further historical information see [99, Notes to Ch. 7]. The basic operation of the RSK algorithm is row insertion, i.e., inserting an integer $i$ into a tableau $T$ with distinct entries and with increasing rows and columns. (Thus $T$ satisfies the conditions of an SYT except that its entries can be any distinct integers, not just $1,2, \ldots, n$.) The process of row inserting $i$ into $T$ produces another tableau, denoted $T \leftarrow i$, with increasing rows and columns. If $S$ is the set of entries of $T$, then $S \cup\{i\}$ is the set of entries of $T \leftarrow i$. We define $T \leftarrow i$ recursively as follows.

- If the first row of $T$ is empty or the largest entry of the first row of $T$ is less than $i$, then insert $i$ at the end of the first row.

- Otherwise, $i$ replaces (or bumps) the smallest element $j$ in the first row satisfying $j>i$. We then insert $j$ into the second row of $T$ by the same procedure.

For further details concerning the definition and basic properties of $T \leftarrow i$ see e.g. [88, Ch. 3], [99, §7.11].

Let $w=a_{1} a_{2} \ldots a_{n} \in \mathfrak{S}_{n}$, and let $\emptyset$ denote the empty tableau. Define

$$
P_{i}=P_{i}(w)=\left(\cdots\left(\left(\emptyset \leftarrow a_{1}\right) \leftarrow a_{2}\right) \leftarrow \cdots \leftarrow a_{i} .\right.
$$

That is, we start with the empty tableau and successively row insert $a_{1}, a_{2}, \ldots, a_{i}$. Set $P=P(w)=P_{n}(w)$. Define $Q_{0}=\emptyset$, and once $Q_{i-1}$ is defined let $Q_{i}=Q_{i}(w)$ be obtained from $Q_{i-1}$ by inserting $i$ (without changing the position of any of the entries of $\left.Q_{i-1}\right)$ so that $Q_{i}$ and $P_{i}$ have the same shape. Set $Q=Q(w)=Q_{n}(w)$, 
and finally define the output of the RSK algorithm applied to $w$ to be the pair $(P, Q)$, denoted $w \stackrel{\text { rsk }}{\longrightarrow}(P, Q)$. For instance, if $w=31542 \in \mathfrak{S}_{5}$, then we obtain

$$
\begin{aligned}
& P_{1}(w)=3, \quad P_{2}(w)=\begin{array}{l}
1 \\
3,
\end{array} P_{3}(w)=3, \\
& P_{4}(w)=\begin{array}{ll}
14 & 4 \\
3 & 5,
\end{array}, \quad P=P_{5}(w)=\begin{array}{ll}
1 & 2 \\
3 & 4 \\
5
\end{array}
\end{aligned}
$$

It follows that

$$
Q=\begin{array}{ll}
13 \\
24 \\
5
\end{array}
$$

Note. By a theorem of Schützenberger [90], [99, §7.13] we have

$$
Q(w)=P\left(w^{-1}\right),
$$

so we could have in fact taken this formula as the definition of $Q(w)$.

If $w \stackrel{\text { rsk }}{\longrightarrow}(P, Q)$ and $P, Q$ have shape $\lambda$, then we also call $\lambda$ the shape of $w$, denoted $\lambda=\operatorname{sh}(w)$. The conjugate partition $\lambda^{\prime}=\left(\lambda_{1}^{\prime}, \lambda_{2}^{\prime}, \ldots\right)$ of $\lambda$ is the partition whose diagram is the transpose of the diagram of $\lambda$. Equivalently, $j$ occurs exactly $\lambda_{j}-\lambda_{j+1}$ times as a part of $\lambda^{\prime}$. The length $\ell(\lambda)$ is the number of (nonzero) parts of $\lambda$, so $\ell(\lambda)=\lambda_{1}^{\prime}$. The fundamental result of Schensted [89] connecting RSK with increasing and decreasing subsequences is the following.

Theorem 2. Let $w \in \mathfrak{S}_{n}$, and suppose that $\operatorname{sh}(w)=\lambda$. Then

$$
\begin{aligned}
\operatorname{is}(w) & =\lambda_{1}, \\
\operatorname{ds}(w) & =\lambda_{1}^{\prime} .
\end{aligned}
$$

Equation (5) is easy to prove by induction since we need only analyze the effect of the RSK algorithm on the first row of the $P_{i}$ 's. On the other hand, equation (6) is based on the following symmetry property of RSK proved by Schensted. If $w=a_{1} a_{2} \ldots a_{n}$ then let $w^{r}=a_{n} \ldots a_{2} a_{1}$, the reverse of $w$. We then have

$$
w \stackrel{\text { rsk }}{\longrightarrow}(P, Q) \Longrightarrow w^{r} \stackrel{\text { rsk }}{\longrightarrow}\left(P^{t}, \operatorname{evac}(Q)^{t}\right),
$$

where ${ }^{t}$ denotes transpose and $\operatorname{evac}(Q)$ is a certain tableau called the evacuation of $Q$ (first defined by Schützenberger [91]) which we will not define here. Equation (7) shows that if $\operatorname{sh}(w)=\lambda$, then $\operatorname{sh}\left(w^{r}\right)=\lambda^{\prime}$. Since clearly is $\left(w^{r}\right)=\operatorname{ds}(w)$, equation (6) follows from (5).

Theorem 2 has several immediate consequences. The first corollary is the ErdősSzekeres theorem (Theorem 1), for if $\operatorname{sh}(w)=\lambda$, is $(w) \leq p$, and $\mathrm{ds}(w) \leq q$, then $\lambda_{1} \leq p$ and $\lambda_{1}^{\prime} \leq q$. Thus the diagram of $\lambda$ is contained in a $q \times p$ rectangle, so $|\lambda| \leq p q$. By the same token we get a quantitative statement that Theorem 1 is best possible. 
Corollary 3. The number of permutations $w \in \mathfrak{S}_{p q}$, where say $p \leq q$, satisfying is $(w)=p$ and $\operatorname{ds}(w)=q$ is given by

$$
\left(f^{\left(p^{q}\right)}\right)^{2}=\left(\frac{(p q) !}{1^{1} 2^{2} \ldots p^{p}(p+1)^{p} \ldots q^{p}(q+1)^{p-1} \ldots(p+q-1)^{1}}\right)^{2},
$$

where $\left(p^{q}\right)$ denotes the partition with q parts equal to $p$.

Proof. Let $\lambda=\operatorname{sh}(w)$. If is $(w)=p$ and $\operatorname{ds}(w)=q$, then $\lambda_{1}=p$ and $\lambda_{1}^{\prime}=q$. Since $\lambda \vdash p q$, we must have $\lambda=\left(p^{q}\right)$. The number of $v \in \mathfrak{S}_{n}$ with a fixed shape $\mu$ is just $\left(f^{\mu}\right)^{2}$, the number of pairs $(P, Q)$ of SYT of shape $\mu$. Hence the left-hand side of equation (8) follows. The right-hand side is then a consequence of the hook-length formula (3).

An interesting result concerning the extremal permutations in the case $p=q$ in Corollary 3 was obtained by Romik [85, Thm. 5]. It can be stated informally as follows. Pick a random permutation $w \in \mathfrak{S}_{p^{2}}$ satisfying is $(w)=\operatorname{ds}(w)=p$. Let $P_{w}$ be the $p^{2} \times p^{2}$ permutation matrix corresponding to $w$, drawn in the plane so that its corners occupy the points $( \pm 1, \pm 1)$. Then almost surely as $p \rightarrow \infty$ the limiting curve enclosing most of the 1's in $P_{w}$ is given by

$$
\left\{(x, y) \in \mathbb{R}^{2}:\left(x^{2}-y^{2}\right)^{2}+2\left(x^{2}+y^{2}\right)=3\right\} .
$$

See Figure 1. In particular, this curve encloses a fraction $\alpha=0.94545962 \ldots$ of the entire square with vertices $( \pm 1, \pm 1)$. The number $\alpha$ can be expressed in terms of elliptic integrals as

$$
\alpha=2 \int_{0}^{1} \frac{1}{\sqrt{\left(1-t^{2}\right)\left(1-(t / 3)^{2}\right)}} d t-\frac{3}{2} \int_{0}^{1} \sqrt{\frac{1-(t / 3)^{2}}{1-t^{2}}} d t .
$$

Compare with the case of any $w \in \mathfrak{S}_{n}$, when clearly the limiting curve encloses the entire square with vertices $( \pm 1, \pm 1)$. For further information related to permutations $w \in \mathfrak{S}_{p^{2}}$ satisfying is $(w)=\mathrm{ds}(w)=p$, see the paper [80] of Pittel and Romik.

Clearly Corollary 3 can be extended to give a formula [99, Cor. 7.23.18] for the number

$$
g_{p q}(n)=\#\left\{w \in \mathfrak{S}_{n}: \text { is }(w)=p, \operatorname{ds}(w)=q\right\},
$$

namely,

$$
g_{p q}(n)=\sum_{\substack{\lambda \vdash n \\ \lambda_{1}=p, \lambda_{1}^{\prime}=q}}\left(f^{\lambda}\right)^{2}
$$

The usefulness of this formula may not be readily apparent, but Theorem 7 below is an example of its utility. 


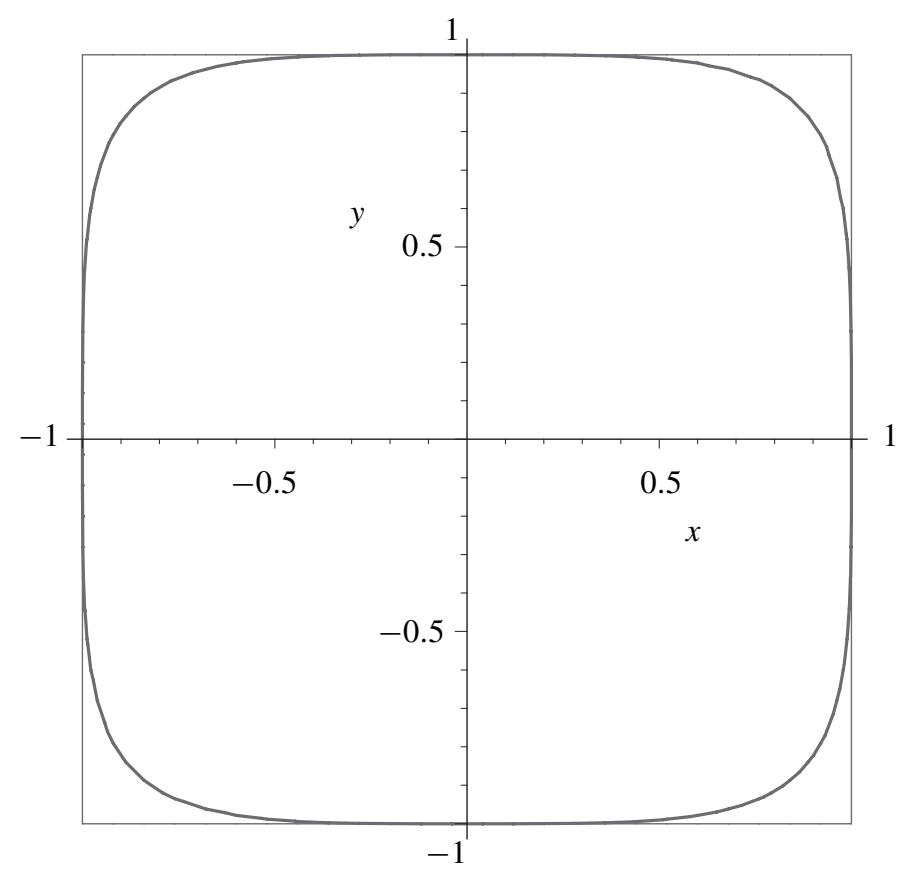

Figure 1. The curve $\left(x^{2}-y^{2}\right)^{2}+2\left(x^{2}+y^{2}\right)=3$.

\section{Expectation of is $(w)$}

A further application of Theorem 2 concerns the distribution of the function is $(w)$ as $w$ ranges over $\mathfrak{S}_{n}$. The problem of obtaining information on this distribution was first raised by Ulam $[109, \S 11.3]$ in the context of Monte Carlo calculations. In particular, one can ask for information on the expectation $E(n)$ of is $(w)$ for $w \in \mathfrak{S}_{n}$, i.e.,

$$
E(n)=\frac{1}{n !} \sum_{w \in \mathfrak{S}_{n}} \text { is }(w) .
$$

Ulam mentions the computations of E. Neighbor suggesting that $E(n)$ is about $1.7 \sqrt{n}$. Numerical experiments by Baer and Brock [14] suggested that $E(n) \sim 2 \sqrt{n}$ might be closer to the truth. The Erdős-Szekeres theorem (Theorem 1) implies immediately that $E(n) \geq \sqrt{n}$, since

$$
\frac{1}{2}\left(\operatorname{is}(w)+\operatorname{is}\left(w^{r}\right)\right) \geq \sqrt{\operatorname{is}(w) \text { is }\left(w^{r}\right)}=\sqrt{\operatorname{is}(w) \operatorname{ds}(w)} \geq \sqrt{n}
$$

Hammersley [56] was the first person to seriously consider the question of estimating $E(n)$. He showed that if

$$
c=\lim _{n \rightarrow \infty} \frac{E(n)}{\sqrt{n}}
$$


then $c$ exists and satisfies

$$
\frac{\pi}{2} \leq c \leq e .
$$

He also gave a heuristic argument that $c=2$, in agreement with the experiments of Baer and Brock.

The next progress on Ulam's problem was based on Schensted's theorem (Theorem 2). It follows from this result that

$$
E(n)=\frac{1}{n !} \sum_{\lambda \vdash n} \lambda_{1}\left(f^{\lambda}\right)^{2} .
$$

Now the RSK algorithm itself shows that

$$
n !=\sum_{\lambda \vdash n}\left(f^{\lambda}\right)^{2},
$$

in agreement with (2). Since the number of terms in the sum on the right-hand side of (11) is very small compared to $n$ !, the maximum value of $f^{\lambda}$ for $\lambda \vdash n$ is close to $\sqrt{n !}$. Let $\lambda^{n}$ be a value of $\lambda \vdash n$ for which $f^{\lambda}$ is maximized. Then by (10) we see that a close approximation to $E(n)$ is given by

$$
\begin{aligned}
E(n) & \approx \frac{1}{n !}\left(\lambda^{n}\right)_{1}\left(f^{\lambda^{n}}\right)^{2} \\
& \approx\left(\lambda^{n}\right)_{1} .
\end{aligned}
$$

This heuristic argument shows the importance of determining the partition $\lambda^{n}$ maximizing the value of $f^{\lambda}$ for $\lambda \vdash n$.

We are only really interested in the behavior of $\lambda^{n}$ as $n \rightarrow \infty$, so let us normalize the Young diagram of any partition $\lambda$ to have area one. Thus each square of the diagram has length $1 / \sqrt{n}$. Let the upper boundary of (the diagram of) $\lambda$ be the $y$-axis directed to the right, and the left boundary be the $x$-axis directed down. As $n \rightarrow \infty$ it is not unreasonable to expect that the boundary of the partition $\lambda^{n}$ will approach some limiting curve $y=\Psi(x)$. If this curve intersects the $x$-axis at $x=b$, then it is immediate that

$$
c:=\lim _{n \rightarrow \infty} \frac{E(n)}{\sqrt{n}} \geq b .
$$

We cannot be sure that $b=c$ since conceivably the first few parts of $\lambda^{n}$ are much larger than the other parts, so these parts would "stretch out" the curve $y=\Psi(x)$ along the $x$-axis.

It was shown independently by Vershik-Kerov [110] and Logan-Shepp [71] that $y=\Psi(x)$ indeed does exist and is given parametrically by

$$
\begin{aligned}
& x=y+2 \cos \theta, \\
& y=\frac{2}{\pi}(\sin \theta-\theta \cos \theta),
\end{aligned}
$$


for $0 \leq \theta \leq \pi$. See Figure 2, where we have placed the coordinate axes in their customary locations.

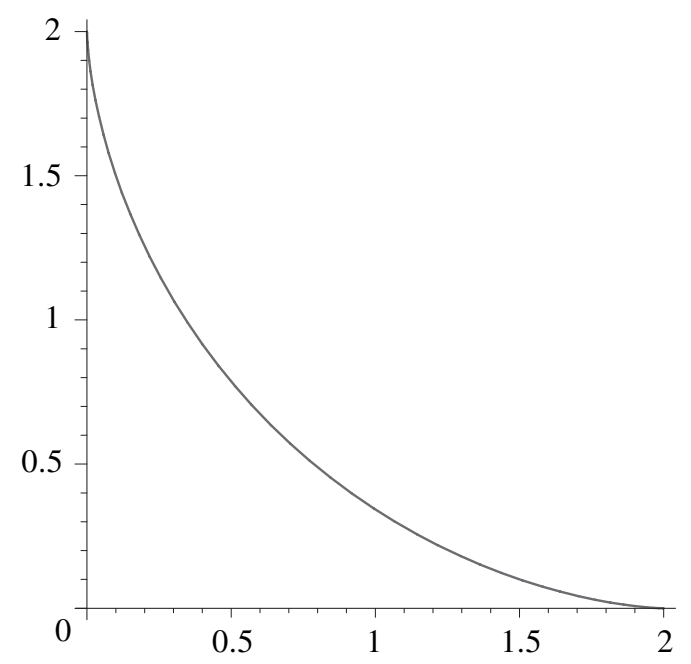

Figure 2. The curve $y=\Psi(x)$.

Logan-Shepp and Vershik-Kerov obtain the curve $\Psi(x)$ as a solution to a variational problem. If $(x, y)$ is a point in the region $A$ enclosed by the curve and the coordinate axes, then the (normalized) hook-length at $(x, y)$ is $f(x)-y+f^{-1}(y)-x$. By equation (3) we maximize $f^{\lambda}$ by minimizing $\prod_{u \in \lambda} h(u)$. Hence in the limit we wish to minimize $I(f)=\iint_{A} \log \left(f(x)-y+f^{-1}(y)-x\right) d x d y$, subject to the normalization $\iint_{A} d x d y=1$. It is shown in [71] and [110] that $f=\Psi$ is the unique function minimizing $I(f)$ (and moreover $I(\Psi)=-1 / 2$ ).

Note. If we extend the curve $y=\Psi(x)$ to include the $x$-axis for $x \geq 2$ and the $y$-axis for $y \geq 2$, and then rotate it $45^{\circ}$ counterclockwise, then we obtain the curve

$$
\Omega(x)= \begin{cases}\frac{2}{\pi}\left(x \arcsin (x / 2)+\sqrt{4-x^{2}}\right), & |x| \leq 2 \\ |x|, & |x| \geq 2\end{cases}
$$

This form of the limiting curve is more convenient for some purposes [62], [63], [64], such as surprising connections with the separation of zeros of orthogonal polynomials.

We see immediately from the equations for $\Psi(x)$ that it intersects the $x$-axis at $x=2$, so $c \geq 2$. By a simple but clever use of the RSK algorithm Vershik and Kerov show in their paper that $c \leq 2$, so we conclude that

$$
E(n) \sim 2 \sqrt{n} .
$$


Different proofs that $E(n) \sim 2 \sqrt{n}$ were later given by Aldous and Diaconis [4], Groeneboom [51], Johansson [58], and Sepäläinen [93]. The proof of Aldous and Diaconis is based on an interacting particle process for which the number of particles remaining after $n$ steps has the same distribution as is ${ }_{n}$. Their proof is known in the language of statistical physics as a hydrodynamic limit argument. See [5, §3] for a brief survey.

We should remark that the curve $y=\Psi(x)$ is not merely the limiting curve for the partition maximizing $f^{\lambda}$; it is also the limiting curve for the typical shape of a permutation $w \in \mathfrak{S}_{n}$. A remarkable refinement of this fact is due to Kerov [57], [61], $[64, \S 0.3 .4]$, who shows that the deviation of a Young diagram from the expected limit converges in probability to a certain Gaussian process. A different kind of refinement is due to Borodin, Okounkov, and Olshanski [31, Thm. 1], who obtain more detailed local information about a typical shape $\lambda$ than is given by $\Psi(x)$.

\section{Distribution of is $(w)$}

A major breakthrough in understanding the behavior of is $(w)$ was achieved in 1999 by Baik, Deift, and Johansson [15]. They determined the entire limiting distribution of is $(w)$ as $n \rightarrow \infty$. It turns out to be given by the (suitably scaled) Tracy-Widom distribution, which had first appeared in connection with the distribution of the largest eigenvalue of a random hermitian matrix.

To describe these results, write is $n$ for the function is: $\mathfrak{S}_{n} \rightarrow \mathbb{Z}$. Let $u(x)$ denote the unique solution to the nonlinear second order differential equation

$$
u^{\prime \prime}(x)=2 u(x)^{3}+x u(x)
$$

subject to the condition

$$
u(x) \sim-\frac{e^{-\frac{2}{3} x^{3 / 2}}}{2 \sqrt{\pi} x^{1 / 4}}, \quad \text { as } x \rightarrow \infty .
$$

Equation (13) is known as the Painlevé II equation, after Paul Painlevé (1863-1933). Painlevé completely classified differential equations (from a certain class of second order equations) whose "bad" singularities (branch points and essential singularities) were independent of the initial conditions. Most of the equations in this class were already known, but a few were new, including equation (13).

Now define the Tracy-Widom distribution to be the probability distribution on $\mathbb{R}$ given by

$$
F(t)=\exp \left(-\int_{t}^{\infty}(x-t) u(x)^{2} d x\right)
$$

It is easily seen that $F(t)$ is indeed a probability distribution, i.e., $F(t) \geq 0$ and $\int_{-\infty}^{\infty} F^{\prime}(t) d t=1$. We can now state the remarkable results of Baik, Deift, and Johansson. 
Theorem 4. We have for random (uniform) $w \in \mathfrak{S}_{n}$ and all $t \in \mathbb{R}$ that

$$
\lim _{n \rightarrow \infty} \operatorname{Prob}\left(\frac{\operatorname{is}_{n}(w)-2 \sqrt{n}}{n^{1 / 6}} \leq t\right)=F(t) .
$$

The above theorem is a vast refinement of the Vershik-Kerov and Logan-Shepp results concerning $E(n)$, the expectation of is $(w)$. It gives the entire limiting distribution (as $n \rightarrow \infty$ ) of is s $_{n}(w)$. Baik, Deift, and Johansson also determine all the limiting moments of is $_{n}(w)$. In particular, we have the following formula for the variance $\operatorname{Var}\left(\right.$ is $\left._{n}\right)$ of is as $_{n} \rightarrow \infty$.

Corollary 5. We have

$$
\begin{aligned}
\lim _{n \rightarrow \infty} \frac{\operatorname{Var}\left(\mathrm{is}_{n}\right)}{n^{1 / 3}} & =\int t^{2} d F(t)-\left(\int t d F(t)\right)^{2} \\
& =0.8131947928 \ldots,
\end{aligned}
$$

and

$$
\begin{aligned}
\lim _{n \rightarrow \infty} \frac{E(n)-2 \sqrt{n}}{n^{1 / 6}} & =\int t d F(t) \\
& =-1.7710868074 \ldots
\end{aligned}
$$

Note that equation (15) may be rewritten

$$
E(n)=2 \sqrt{n}+\alpha n^{1 / 6}+o\left(n^{1 / 6}\right),
$$

where $\alpha=\int t d F(t)$, thereby giving the second term in the asymptotic behavior of $E(n)$.

We will say only a brief word on the proof of Theorem 4, explaining how combinatorics enters into the picture. Some kind of analytic expression is needed for the distribution of $\operatorname{is}_{n}(w)$. Such an expression is provided by the following result of Ira Gessel [47], later proved in other ways by various persons; see [15, §1] for references. Define

$$
\begin{aligned}
u_{k}(n) & =\#\left\{w \in \mathfrak{S}_{n}: \operatorname{is}_{n}(w) \leq k\right\}, \\
U_{k}(x) & =\sum_{n \geq 0} u_{k}(n) \frac{x^{2 n}}{n !^{2}}, \quad k \geq 1, \\
I_{i}(2 x) & =\sum_{n \geq 0} \frac{x^{2 n+i}}{n !(n+i) !}, \quad i \in \mathbb{Z} .
\end{aligned}
$$

The function $I_{i}$ is the hyperbolic Bessel function of the first kind of order $i$. Note that $I_{i}(2 x)=I_{-i}(2 x)$. 
Theorem 6. We have

$$
U_{k}(x)=\operatorname{det}\left(I_{i-j}(2 x)\right)_{i, j=1}^{k} .
$$

Example 1. We have (using $I_{1}=I_{-1}$ )

$$
U_{2}(x)=\left|\begin{array}{ll}
I_{0}(2 x) & I_{1}(2 x) \\
I_{1}(2 x) & I_{0}(2 x)
\end{array}\right|=I_{0}(2 x)^{2}-I_{1}(2 x)^{2} .
$$

From this expression it is easy to deduce that

$$
u_{2}(n)=\frac{1}{n+1}\left(\begin{array}{c}
2 n \\
n
\end{array}\right),
$$

a Catalan number. This formula for $u_{2}(n)$ was first stated by Hammersley [56] in 1972, with the first published proofs by Knuth [67, §5.1.4] and Rotem [86]. There is a more complicated expression for $u_{3}(n)$ due to Gessel [47, §7], [99, Exer. 7.16(e)], namely,

$$
u_{3}(n)=\frac{1}{(n+1)^{2}(n+2)} \sum_{j=0}^{n}\left(\begin{array}{c}
2 j \\
j
\end{array}\right)\left(\begin{array}{l}
n+1 \\
j+1
\end{array}\right)\left(\begin{array}{l}
n+2 \\
j+2
\end{array}\right),
$$

while no "nice" formula for $u_{k}(n)$ is known for fixed $k>3$. It is known, however, that $u_{k}(n)$ is a P-recursive function of $n$, i.e., satisfies a linear recurrence with polynomial coefficients $[47, \S 7]$. For instance,

$$
\begin{aligned}
(n+4)(n+3)^{2} u_{4}(n)=( & \left.20 n^{3}+62 n^{2}+22 n-24\right) u_{4}(n-1) \\
& -64 n(n-1)^{2} u_{4}(n-2), \\
(n+6)^{2}(n+4)^{2} u_{5}(n)=( & \left.375-400 n-843 n^{2}-322 n^{3}-35 n^{4}\right) u_{5}(n-1) \\
+ & \left(259 n^{2}+622 n+45\right)(n-1)^{2} u_{5}(n-2) \\
- & 225(n-1)^{2}(n-2)^{2} u_{5}(n-3) .
\end{aligned}
$$

A number of conjectures about the form of the recurrence satisfied by $u_{k}(n)$ were made by Bergeron, Favreau, and Krob [23], reformulated in [24] with some progress toward a proof.

Gessel's theorem (Theorem 6) reduces the theorem of Baik, Deift, and Johansson to "just" analysis, viz., the Riemann-Hilbert problem in the theory of integrable systems, followed by the method of steepest descent to analyze the asymptotic behavior of integrable systems. For further information see the survey [40] of Deift mentioned above.

The asymptotic behavior of is ${ }_{n}(w)$ (suitably scaled) turned out to be identical to the Tracy-Widom distribution $F(t)$ of equation (14). Originally the Tracy-Widom distribution arose in connection with the Gaussian Unitary Ensemble (GUE). GUE is 
a certain natural probability density on the space of all $n \times n$ hermitian matrices $M=\left(M_{i j}\right)$, namely,

$$
Z_{n}^{-1} e^{-\operatorname{tr}\left(M^{2}\right)} d M
$$

where $Z_{n}$ is a normalization constant and

$$
d M=\prod_{i} d M_{i i} \cdot \prod_{i<j} d\left(\operatorname{Re} M_{i j}\right) d\left(\operatorname{Im} M_{i j}\right)
$$

Let the eigenvalues of $M$ be $\alpha_{1} \geq \alpha_{2} \geq \cdots \geq \alpha_{n}$. The following result marked the eponymous appearance [106] of the Tracy-Widom distribution:

$$
\lim _{n \rightarrow \infty} \operatorname{Prob}\left(\left(\alpha_{1}-\sqrt{2 n}\right) \sqrt{2} n^{1 / 6} \leq t\right)=F(t) .
$$

Thus as $n \rightarrow \infty$, is $_{n}(w)$ and $\alpha_{1}$ have the same distribution (after scaling).

It is natural to ask, firstly, whether there is a result analogous to equation (19) for the other eigenvalues $\alpha_{k}$ of the GUE matrix $M$, and, secondly, whether there is some connection between such a result and the behavior of increasing subsequences of random permutations. A generalization of (19) to all $\alpha_{k}$ was given by Tracy and Widom [106] (expressed in terms of the Painlevé II function $u(x)$ ). The connection with increasing subsequences was conjectured in [15] and proved independently by Borodin-Okounkov-Olshanski [31], Johannson [59], and Okounkov [78], after first being proved for the second largest eigenvalue by Baik, Deift, and Johansson [16]. Given $w \in \mathfrak{S}_{n}$, define integers $\lambda_{1}, \lambda_{2}, \ldots$ by letting $\lambda_{1}+\cdots+\lambda_{k}$ be the largest number of elements in the union of $k$ increasing subsequences of $w$. For instance, let $w=247951368$. The longest increasing subsequence is 24568 , so $\lambda_{1}=5$. The largest union of two increasing subsequences is 24791368 (the union of 2479 and 1368), so $\lambda_{1}+\lambda_{2}=8$. (Note that it is impossible to find a union of length 8 of two increasing subsequences that contains an increasing subsequence of length $\lambda_{1}=5$.) Finally $w$ itself is the union of the three increasing subsequences 2479, 1368, and 5, so $\lambda_{1}+\lambda_{2}+\lambda_{3}=9$. Hence $\left(\lambda_{1}, \lambda_{2}, \lambda_{3}\right)=(5,3,1)$ (and $\lambda_{i}=0$ for $i>3$ ). Readers familiar with the theory of the RSK algorithm will recognize the sequence $\left(\lambda_{1}, \lambda_{2}, \ldots\right)$ as the shape $\operatorname{sh}(w)$ as defined preceding Theorem 2, a well-known result of Curtis Greene [50], [99, Thm. A1.1.1]. (In particular, $\lambda_{1} \geq \lambda_{2} \geq \cdots$, a fact which is by no means obvious.) The result of [31], [59], [78] asserts that as $n \rightarrow \infty, \lambda_{k}$ and $\alpha_{k}$ are equidistributed, up to scaling. In particular, the paper [78] of Okounkov provides a direct connection, via the topology of random surfaces, between the two seemingly unrelated appearances of the Tracy-Widom distribution in the theories of random matrices and increasing subsequences. A very brief explanation of this connection is the following: a surface can be described either by gluing together polygons along their edges or by a ramified covering of a sphere. The former description is related to random matrices via the theory of quantum gravity, while the latter can be formulated in terms of the combinatorics of permutations. 
We have discussed how Gessel's generating function $U_{k}(x)$ for $u_{k}(n)$ is needed to find the limiting distribution of is ${ }_{n}$. We can also ask about the behavior of $u_{k}(n)$ itself for fixed $k$. The main result here is due to Regev [82].

Theorem 7. For fixed $k$ and for $n \rightarrow \infty$ we have the asymptotic formula

$$
u_{k}(n) \sim 1 ! 2 ! \ldots(k-1) !\left(\frac{1}{\sqrt{2 \pi}}\right)^{k-1}\left(\frac{1}{2}\right)^{\left(k^{2}-1\right) / 2} k^{k^{2} / 2} \frac{k^{2 n}}{n^{\left(k^{2}-1\right) / 2}} .
$$

Idea of proof. From the RSK algorithm we have

$$
u_{k}(n)=\sum_{\substack{\lambda \vdash n \\ \ell(\lambda) \leq k}}\left(f^{\lambda}\right)^{2}
$$

Write $f^{\lambda}$ in terms of the hook-length formula, factor out the dominant term from the sum (which can be determined via Stirling's formula), and interpret what remains in the limit $n \rightarrow \infty$ as a $k$-dimensional integral. This integral turns out to be a special case of Selberg's integral (e.g., [7, Ch. 8]), which can be explicitly evaluated.

An immediate corollary of Theorem 7 (which can also be easily proved directly using RSK) is the formula

$$
\lim _{n \rightarrow \infty} u_{k}(n)^{1 / n}=k^{2}
$$

\section{Symmetry}

Previous sections dealt with properties of general permutations in $\mathfrak{S}_{n}$. Much of the theory carries over for certain classes of permutations. There is a natural action of the dihedral group $D_{4}$ of order 8 on $\mathfrak{S}_{n}$, best understood by considering the permutation matrix $P_{w}$ corresponding to $w \in \mathfrak{S}_{n}$. Since $P_{w}$ is a square matrix, $D_{4}$ acts on $P_{w}$ as the usual symmetry group of the square. In particular, reflecting through the main diagonal transforms $P_{w}$ to its transpose $P_{w}^{t}=P_{w^{-1}}$. Reflecting about a horizontal line produces $P_{w^{r}}$, where $w^{r}$ is the reverse of $w$ as used in equation (7). These two reflections generate the entire group $D_{4}$.

Let $G$ be a subgroup of $D_{4}$, and let

$$
\mathfrak{S}_{n}^{G}=\left\{w \in \mathfrak{S}_{n}: \sigma \cdot w=w \text { for all } \sigma \in G\right\} .
$$

Most of the results of the preceding sections can be carried over from $\mathfrak{S}_{n}$ to $\mathfrak{S}_{n}^{G}$. The general theory is due to Baik and Rains [17], [18], [19]. Moreover, for certain $G$ we can add the condition that no entry of $P_{w}$ equal to 1 can be fixed by $G$, or more strongly we can specify the number of 1's in $P_{w}$ fixed by $G$. For instance, if $G$ is the group of order 2 generated by reflection through the main diagonal, then we are specifying the number of fixed points of $w$. For convenience we will consider here only two special 
cases, viz., (a) $G$ is the group of order 2 generated by reflection through the main diagonal. In this case $\mathfrak{S}_{n}^{G}=\left\{w \in \mathfrak{S}_{n}: w^{2}=1\right\}$, the set of involutions in $\mathfrak{S}_{n}$, which we also denote as $\mathfrak{I}_{n}$. (b) The modification of (a) where we consider fixed-point free involutions only. Write $\mathfrak{I}_{n}^{*}$ for this set, so $\mathfrak{I}_{n}^{*}=\emptyset$ when $n$ is odd.

The RSK algorithm is well-behaved with respect to inversion, viz., it follows from equation (4) that if $w \stackrel{\text { rsk }}{\longrightarrow}(P, Q)$ then $w^{-1} \stackrel{\text { rsk }}{\longrightarrow}(Q, P)$. Hence

$$
w^{2}=1 \quad \text { if and only if } P=Q .
$$

Let

$$
y_{k}(n)=\#\left\{w \in \mathfrak{I}_{n}: \operatorname{is}_{n}(w) \leq k\right\} .
$$

By Schensted's theorem (Theorem 2) we conclude

$$
y_{k}(n)=\sum_{\substack{\lambda \vdash n \\ \lambda_{1} \leq k}} f^{\lambda},
$$

the "involution analogue" of (20). From this formula or by other means one can obtain formulas for $y_{k}(n)$ for small $k$ analogous to (17) and (18). In particular (see [99, Exer. 7.16(b)] for references),

$$
\begin{aligned}
& y_{2}(n)=\left(\begin{array}{c}
n \\
\lfloor n / 2\rfloor
\end{array}\right), \\
& y_{3}(n)=\sum_{i=0}^{\lfloor n / 2\rfloor}\left(\begin{array}{c}
n \\
2 i
\end{array}\right) C_{i}, \\
& y_{4}(n)=C_{\lfloor(n+1) / 2\rfloor} C_{\lceil(n+1) / 2\rceil}, \\
& y_{5}(n)=6 \sum_{i=0}^{\lfloor n / 2\rfloor}\left(\begin{array}{l}
n \\
2 i
\end{array}\right) C_{i} \frac{(2 i+2) !}{(i+2) !(i+3) !},
\end{aligned}
$$

where as usual $C_{i}$ is a Catalan number.

The RSK algorithm is also well-behaved with respect to fixed points of involutions. It was first shown by Schützenberger [90, p. 127], [99, Exer. 7.28(a)] that if $w^{2}=1$ and $w \stackrel{\text { rsk }}{\longrightarrow}(P, P)$, then the number of fixed points of $w$ is equal to the number of columns of $P$ of odd length. Let

$$
\begin{gathered}
v_{2 k}(n)=\#\left\{w \in \mathfrak{I}_{n}^{*}: \operatorname{ds}(w) \leq 2 k\right\}, \\
z_{k}(n)=\#\left\{w \in \mathfrak{I}_{n}^{*}: \text { is }(w) \leq k\right\} .
\end{gathered}
$$

(It is easy to see directly that if $w \in \mathfrak{I}_{n}^{*}$ then ds $(w)$ is even, so there is no need to deal 
with $v_{2 k+1}(n)$.) It follows that

$$
\begin{aligned}
v_{2 k}(n) & =\sum_{\substack{\lambda \vdash n \\
\lambda_{1} \leq k}} f^{2 \lambda^{\prime}}, \\
z_{k}(n) & =\sum_{\substack{\lambda \vdash n, \lambda_{1}^{\prime} \leq k}} f^{2 \lambda^{\prime}},
\end{aligned}
$$

where $2 \lambda^{\prime}=\left(2 \lambda_{1}^{\prime}, 2 \lambda_{2}^{\prime}, \ldots\right)$, the general partition with no columns of odd length. Note that for fixed-point free involutions $w \in \mathfrak{I}_{n}^{*}$ we no longer have a symmetry between is $(w)$ and ds $(w)$, as we do for arbitrary permutations or arbitrary involutions.

There are also "involution analogues" of Gessel's determinant (Theorem 6). Equations (23) and (24) below were first obtained by Gessel [47, §6], equation (25) by Goulden [48], and equations (26) and (27) by Baik and Rains [17, Cor. 5.5]. Let

$$
\begin{aligned}
Y_{k}(x) & =\sum_{n \geq 0} y_{k}(n) \frac{x^{n}}{n !}, \\
V_{2 k}(x) & =\sum_{n \geq 0} v_{2 k}(n) \frac{x^{n}}{n !}, \\
Z_{k}(x) & =\sum_{n \geq 0} z_{k}(n) \frac{x^{n}}{n !} .
\end{aligned}
$$

Write $I_{i}=I_{i}(2 x)$.

Theorem 8. We have

$$
\begin{aligned}
Y_{2 k}(x) & =\operatorname{det}\left(I_{i-j}+I_{i+j-1}\right)_{i, j=1}^{k}, \\
Y_{2 k+1}(x) & =e^{x} \operatorname{det}\left(I_{i-j}-I_{i+j}\right)_{i, j=1}^{k}, \\
V_{2 k}(x) & =\operatorname{det}\left(I_{i-j}-I_{i+j}\right)_{i, j=1}^{k}, \\
Z_{2 k}(x) & =\frac{1}{4} \operatorname{det}\left(I_{i-j}+I_{i+j-2}\right)_{i, j=1}^{k}+\frac{1}{2} \operatorname{det}\left(I_{i-j}-I_{i+j}\right)_{i, j=1}^{k-1}, \\
Z_{2 k+1}(x) & =\frac{1}{2} e^{x} \operatorname{det}\left(I_{i-j}-I_{i+j-1}\right)_{i, j=1}^{k}+\frac{1}{2} e^{-x} \operatorname{det}\left(I_{i-j}+I_{i+j-1}\right)_{i, j=1}^{k} .
\end{aligned}
$$

Once we have the formulas of Theorem 8 we can use the techniques of Baik, Deift, and Johansson to obtain the limiting behavior of $\operatorname{ds}(w)$ for $w \in \mathfrak{I}_{n}$ and $w \in \mathfrak{I}_{n}^{*}$. These results were first obtained by Baik and Rains [18], [19].

Theorem 9. (a) We have for random (uniform) $w \in \mathfrak{I}_{n}$ and all $t \in \mathbb{R}$ that

$$
\lim _{n \rightarrow \infty} \operatorname{Prob}\left(\frac{\operatorname{is}_{n}(w)-2 \sqrt{n}}{n^{1 / 6}} \leq t\right)=F(t)^{1 / 2} \exp \left(\frac{1}{2} \int_{t}^{\infty} u(s) d s\right),
$$


where $F(t)$ denotes the Tracy-Widom distribution and $u(s)$ the Painlevé II function. (By (22) we can replace is ${ }_{n}(w)$ with $\mathrm{ds}_{n}(w)$.)

(b) We have for random (uniform) $w \in \mathfrak{I}_{2 n}^{*}$ and all $t \in \mathbb{R}$ that

$$
\lim _{n \rightarrow \infty} \operatorname{Prob}\left(\frac{\mathrm{ds}_{2 n}(w)-2 \sqrt{2 n}}{(2 n)^{1 / 6}} \leq t\right)=F(t)^{1 / 2} \exp \left(\frac{1}{2} \int_{t}^{\infty} u(s) d s\right) .
$$

(c) We have for random (uniform) $w \in \mathfrak{I}_{2 n}^{*}$ and all $t \in \mathbb{R}$ that

$$
\lim _{n \rightarrow \infty} \operatorname{Prob}\left(\frac{\operatorname{is}_{2 n}(w)-2 \sqrt{2 n}}{(2 n)^{1 / 6}} \leq t\right)=F(t)^{1 / 2} \cosh \left(\frac{1}{2} \int_{t}^{\infty} u(s) d s\right) .
$$

There are orthogonal and symplectic analogues of the GUE model of random hermitian matrices, known as the GOE and GSE models. The GOE model replaces hermitian matrices with real symmetric matrices, while the GSE model concerns hermitian self-dual matrices. (A $2 n \times 2 n$ complex matrix is hermitian self-dual if is composed of $2 \times 2$ blocks of the form $\left[\begin{array}{cc}a+b i & c+d i \\ -c+d i & a-b i\end{array}\right]$ which we identify with the quaternion $a+b i+c j+d k$, such that if we regard the matrix as an $n \times n$ matrix $M$ of quaternions, then $\bar{M}_{j i}=M_{i j}$ where the bar is quaternion conjugation.) The limiting distribution of $\operatorname{ds}_{2 n}(w)$ for $w \in \mathfrak{I}_{2 n}^{*}$ coincides (after scaling) with the distribution of the largest eigenvalue of a random real symmetric matrix (under the GOE model), while the limiting distribution of is $2 n(w)$ for $w \in \mathfrak{I}_{2 n}^{*}$ coincides (after scaling) with the distribution of the largest eigenvalue of a random hermitian self-dual matrix (under the GSE model) [107].

\section{Connections with the classical groups}

In equation (9) we expressed $g_{p q}(n)$, the number of permutations $w \in \mathfrak{S}_{n}$ satisfying is $(w)=p$ and $\operatorname{ds}(w)=q$, in terms of the degrees $f^{\lambda}$ of irreducible representations of $\mathfrak{S}_{n}$. This result can be restated via Schur-Weyl duality as a statement about the distribution of eigenvalues of matrices in the unitary group $U(n)$. The results of Section 5 can be used to extend this statement to other classical groups.

Let $U(k)$ denote the group of $k \times k$ complex unitary matrices. For a function $f: U(k) \rightarrow \mathbb{C}$, let $E(f)$ denote expectation with respect to Haar measure, i.e.,

$$
E(f)=\int_{M \in U(k)} f(M) d M
$$

where $\int$ is the Haar integral. The following result was proved by Diaconis and Shahshahani [42] for $n \geq k$ and by Rains [81] for general $k$. Note that if $M$ has eigenvalues $\theta_{1}, \ldots, \theta_{k}$ then

$$
\left|\operatorname{tr}(M)^{n}\right|^{2}=\left(\theta_{1}+\cdots+\theta_{k}\right)^{n}\left(\bar{\theta}_{1}+\cdots+\bar{\theta}_{k}\right)^{n} .
$$


Theorem 10. We have $E\left(\left|\operatorname{tr}(M)^{n}\right|^{2}\right)=u_{k}(n)$, where $u_{k}(n)$ is defined in equation (16).

Proof. The proof is based on the theory of symmetric functions, as developed e.g. in [72] or [99, Ch. 7]. If $f\left(x_{1}, \ldots, x_{k}\right)$ is a symmetric function, then write $f(M)$ for $f\left(\theta_{1}, \ldots, \theta_{k}\right)$, where $\theta_{1}, \ldots, \theta_{k}$ are the eigenvalues of $M \in U(k)$. The Schur functions $s_{\lambda}$ for $\ell(\lambda) \leq k$ are the irreducible characters of $U(k)$, so by the orthogonality of characters we have for partitions $\lambda, \mu$ of length at most $k$ that

$$
\int_{M \in U(k)} s_{\lambda}(M) \overline{s_{\mu}(M)} d M=\delta_{\lambda \mu}
$$

Now $\operatorname{tr}(M)^{n}=p_{1}(M)^{n}$, where $p_{1}\left(x_{1}, \ldots, x_{k}\right)=x_{1}+\cdots+x_{k}$. The symmetric function $p_{1}\left(x_{1}, \ldots, x_{k}\right)^{n}$ has the expansion [72, Exam. 1.5.2], [99, Cor. 7.12.5]

$$
p_{1}\left(x_{1}, \ldots, x_{k}\right)^{n}=\sum_{\substack{\lambda \vdash n \\ \ell(\lambda) \leq k}} f^{\lambda} s_{\lambda}\left(x_{1}, \ldots, x_{k}\right),
$$

where $f^{\lambda}$ is the number of SYT of shape $\lambda$ as in Section 2. (This formula is best understood algebraically as a consequence of the Schur-Weyl duality between $\mathfrak{S}_{n}$ and $U(k)$ [99, Ch. 7, Appendix 2], although it can be proved without any recourse to representation theory.) Hence from equation (28) we obtain

$$
\begin{aligned}
E\left(\left|\operatorname{tr}(M)^{n}\right|^{2}\right) & =\int_{\substack{M \in U(k)\\
}} p_{1}(M)^{n}{\overline{p_{1}(M)}}^{n} d M \\
& =\sum_{\substack{\lambda \vdash n \\
\ell(\lambda) \leq k}}\left(f^{\lambda}\right)^{2} .
\end{aligned}
$$

Comparing with equation (20) completes the proof.

Many variations of Theorem 10 have been investigated. For instance, we can replace $\operatorname{tr}(M)^{n}$ by more general symmetric functions of the eigenvalues, such as $\operatorname{tr}\left(M^{m}\right)^{n}$, or we can replace $U(k)$ with other classical groups, i.e., $O(k)$ and $\operatorname{Sp}(2 k)$. For further information, see Rains [81].

\section{Pattern avoidance}

In this and the following two sections we consider some generalizations of increasing and decreasing subsequences of permutations. In this section and the next we look at other kinds of subsequences of permutations, while in Section 9 we generalize the permutations themselves.

We have defined $u_{k}(n)$ to be the number of permutations in $\mathfrak{S}_{n}$ with no increasing subsequence of length $k+1$. We can instead prohibit other types of subsequences of a fixed length, leading to the currently very active area of pattern avoidance. 
Given $v=b_{1} \ldots b_{k} \in \mathfrak{S}_{k}$, we say that a permutation $w=a_{1} \ldots a_{n} \in \mathfrak{S}_{n}$ avoids $v$ if it contains no subsequence $a_{i_{1}} \ldots a_{i_{k}}$ in the same relative order as $v$, i.e., no subsequence $a_{i_{1}} \ldots a_{i_{k}}$ satisfies the condition:

$$
\text { For all } 1 \leq r<s \leq k, a_{i_{r}}<a_{i_{s}} \Longleftrightarrow b_{r}<b_{s} .
$$

Thus a permutation $w$ satisfies is $(w)<k$ if and only if it is $12 \ldots k$-avoiding, and similarly satisfies ds $(w)<k$ if and only if it is $k(k-1) \ldots 1$-avoiding. What can be said about the set $\mathfrak{S}_{n}(v)$ of permutations $w \in \mathfrak{S}_{n}$ that are $v$-avoiding? In particular, when are there formulas and recurrences for $s_{n}(v):=\# \mathfrak{S}_{n}(v)$ similar to those of Theorem 6 and Example 1?

The vast subject of pattern avoidance, as a generalization of avoiding long increasing and decreasing subsequences, began in 1968 with Knuth [66, Exer. 2.2.1.5]. He showed in connection with a problem on stack sorting that $s_{n}(312)$ is the Catalan number $C_{n}$. (See also [99, Exer. 6.19(ff)].) By obvious symmetries this result, together with equation (17), shows that $s_{n}(v)=C_{n}$ for all $v \in \mathfrak{S}_{3}$. A fundamental paper directly connecting 321-avoiding and 231-avoiding permutations was written by Simion and Schmidt [94].

Wilf first raised the question of investigating $s_{n}(v)$ for $v \in \mathfrak{S}_{k}$ when $k \geq 4$. Here is a brief summary of some highlights in this burgeoning area. For further information, see e.g. [30, Chs. 4, 5] and the special issue [9]. Call two permutations $u, v \in \mathfrak{S}_{k}$ equivalent, denoted $u \sim v$, if $s_{n}(u)=s_{n}(v)$ for all $n$. Then there are exactly three equivalence classes of permutations $u \in \mathfrak{S}_{4}$. One class contains 1234, 1243, and 2143 (and their trivial symmetries), the second contains 3142 and 1342, and the third 1324. The values of $s_{n}(1234)$ are given by equation (18) and of $s_{n}(1342)$ are given by the generating function

$$
\sum_{n \geq 0} s_{n}(1342) x^{n}=\frac{32 x}{1+20 x-8 x^{2}-(1-8 x)^{3 / 2}},
$$

a result of Bóna [28].The enumeration of 1324-avoiding permutations in $\mathfrak{S}_{n}$ remains open.

Let us mention one useful technique for showing the equivalence of permutations in $\mathfrak{S}_{k}$, the method of generating trees introduced by Chung, Graham, Hoggatt and Kleiman [38] and further developed by West [111], [112], [113] and others. Given $u \in \mathfrak{S}_{k}$, the generating tree $\mathcal{T}_{u}$ is the tree with vertex set $\bigcup_{n \geq 1} \mathfrak{S}_{n}(u)$, and with $y$ a descendent of $w$ if $w$ is a subsequence of $y$ (an actual subsequence, not the pattern of a subsequence). For many pairs $u, v \in \mathfrak{S}_{k}$ we have $\mathcal{T}_{u} \cong \mathcal{T}_{v}$, showing in particular that $s_{n}(u)=s_{n}(v)$ for all $n$, i.e., $u \sim v$. In many cases in fact the two trees will have no automorphisms, so the isomorphism $\mathcal{T}_{u} \rightarrow \mathcal{T}_{v}$ is unique, yielding a canonical bijection $\mathfrak{S}_{n}(u) \rightarrow \mathfrak{S}_{n}(v)$. A unique isomorphism holds for instance when $u=123$ and $v=132$. Figure 3 shows the first four levels of the trees $\mathcal{T}_{123} \cong \mathcal{T}_{132}$, labelled by elements of both $\mathcal{T}_{123}$ and $\mathcal{T}_{132}$ (boldface). This tree can also be defined recursively by the condition that the root has two children, and if vertex $x$ has $k$ children, then 
the children of $x$ have $2,3, \ldots, k+1$ children. For further information about trees defined in a similar recursive manner, see Banderier et al. [20].

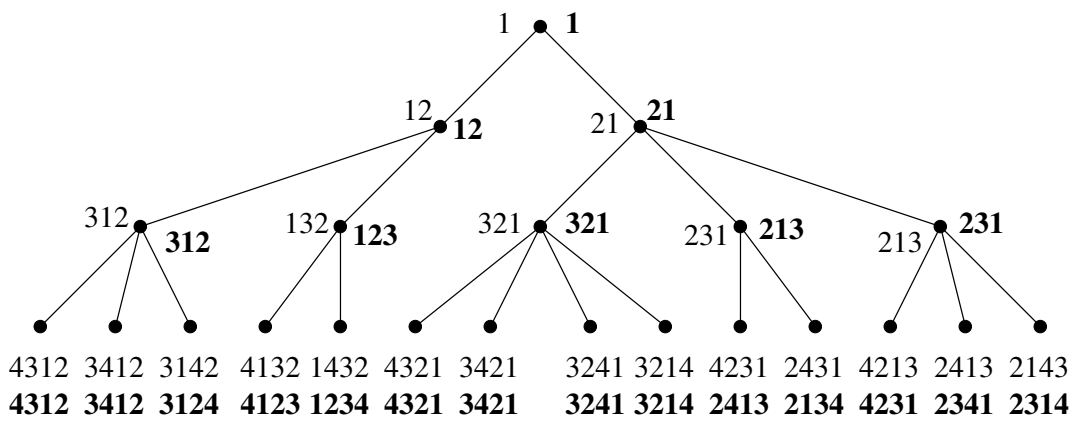

Figure 3. The generating tree for $\mathfrak{S}_{n}(123)$ and $\mathfrak{S}_{n}(132)$.

Given $v \in \mathfrak{S}_{k}$, let

$$
F_{v}(x)=\sum_{n \geq 0} s_{n}(v) x^{n}
$$

It is not known whether $F_{v}(x)$ is always algebraic or even the weaker condition of being $D$-finite, which is equivalent to $s_{n}(v)$ being P-recursive [96], [99, §6.4]. A longstanding conjecture, known as the Stanley-Wilf conjecture, stated that for all $v \in \mathfrak{S}_{k}$ there is a $c>1$ such that $s_{n}(v)<c^{n}$. In this case $L_{v}:=\lim _{n \rightarrow \infty} s_{n}(v)^{1 / n}$ exists and satisfies $1<L_{v}<\infty$ [8]. For instance, equation (21) asserts that $L_{12 \ldots k}=(k-1)^{2}$. The Stanley-Wilf conjecture was proved by Marcus and Tardos [76] by a surprisingly simple argument. It is known that for $k \geq 4$ the permutation $12 \ldots k$ neither maximizes nor minimizes $L_{v}$ for $v \in \mathfrak{S}_{k}$ [3], but it is not known which permutations do achieve the maximum or minimum.

An interesting aspect of pattern avoidance was considered by Albert [1]. Let $X$ be a finite subset of $\mathfrak{S}_{2} \cup \mathfrak{S}_{3} \cup \cdots$, and let $s_{n}(X)$ denote the number of permutations $w \in \mathfrak{S}_{n}$ avoiding all permutations $v \in X$. We say that $X$ is proper if it does not contain both the identity permutation $12 \ldots j$ for some $j$ and the "reverse identity" $k \ldots 21$ for some $k$. It is easy to see (using Theorem 1) that $X$ is proper if and only if $s_{n}(X)>0$ for all $n \geq 1$. For $w \in \mathfrak{S}_{n}$ let is ${ }^{X}(w)$ be the length of the longest subsequence of $w$ avoiding all $v \in X$, and let $E_{X}(n)$ denote the expectation of is ${ }^{X}(w)$ for uniform $w \in \mathfrak{S}_{n}$, so

$$
E_{X}(n)=\frac{1}{n !} \sum_{w \in \mathfrak{S}_{n}} \text { is }^{X}(w) .
$$

Albert then makes the following intriguing conjecture.

Conjecture 11. If $X$ is proper then

$$
\lim _{n \rightarrow \infty} s_{n}(X)^{1 / n}=\frac{1}{4}\left(\lim _{n \rightarrow \infty} \frac{E_{X}(n)}{\sqrt{n}}\right)^{2} .
$$


(The limits on both sides are only conjectured to exist.)

Consider for instance the case $X=\{21\}$. Then $s_{n}(X)=1$ for all $n \geq 1$, so the left-hand side of (29) is 1 . On the other hand, is ${ }^{X}(w)=$ is $(w)$, so the right-hand side is also 1 by equation (12). More generally, Albert proves Conjecture 11 for $X=\{12 \ldots k\}$ and hence (by symmetry) for $X=\{k \ldots 21\}$ [1, Prop. 4].

Let us mention that permutations avoiding a certain pattern $v$ or a finite set of patterns have arisen naturally in a variety of contexts. For instance, an elementary result of Tenner [104] asserts that the interval $[0, w]$ in the Bruhat order of $\mathfrak{S}_{n}$ is a boolean algebra if and only if $w$ is 321 and 3412-avoiding, and that the number of such permutations $w$ is the Fibonacci number $F_{2 n-1}$. The Schubert polynomial $\mathfrak{S}_{w}$ is a single monomial if and only if $w$ is 132-avoiding [73, p. 46]. All reduced decompositions of $w \in \mathfrak{S}_{n}$ are connected by the Coxeter relations $s_{i} s_{j}=s_{j} s_{i}$ (i.e., $s_{i} s_{i+1} s_{i}$ does not appear as a factor in any reduced decomposition of $w$ ) if and only if $w$ is 321-avoiding [25, Thm. 2.1]. Vexillary permutations may be defined as those permutations $w$ such that the stable Schubert polynomial $F_{w}$ is a single Schur function or equivalently, whose Schubert polynomial $\mathfrak{S}_{w}$ is a flag Schur function (or multi-Schur function). They turn out to be the same as 2143-avoiding permutations [73, (1.27)(iii), (7.24)(iii)], first enumerated by West [111, Cor. 3.17], [112, Cor. 3.11]. Similarly, permutations $w \in \mathfrak{S}_{n}$ for which the Schubert variety $\Omega_{w}$ in the complete flag variety $\operatorname{GL}(n, \mathbb{C}) / B$ is smooth are those permutations that are 4231 and 3412-avoiding (implicit in Ryan [87], based on earlier work of Lakshmibai, Seshadri, and Deodhar, and explicit in Lakshmibai and Sandhya [69]). The enumeration of such "smooth permutations" in $\mathfrak{S}_{n}$ is due to Haiman [29], [52], [99, Exer. 6.47], viz.,

$$
\sum_{n \geq 0} \mathfrak{S}_{n}(4231,3412) x^{n}=\frac{1}{1-x-\frac{x^{2}}{1-x}\left(\frac{2 x}{1+x-(1-x) C(x)}-1\right)},
$$

where $C(x)=\sum_{n \geq 0} C_{n} x^{n}=(1-\sqrt{1-4 x}) / 2 x$. See Billey and Lakshmibai [26] for further information. As a final more complicated example, Billey and Warrington [27] show that a permutation $w \in \mathfrak{S}_{n}$ has a number of nice properties related to the Kazhdan-Lusztig polynomials $P_{x, w}$ if and only if $w$ avoids $321,46718235,46781235$, 56718234, and 56781234. These permutations were later enumerated by Stankova and West [95]. A database of "natural occurrences" of pattern avoidance can be found at a website [105] maintained by B. Tenner.

The subjects of pattern avoidance and increasing/decreasing subsequences can be considered together, by asking for the distribution of is $(w)$ or $\operatorname{ds}(w)$ where $w$ ranges over a pattern-avoiding class $\mathfrak{S}_{n}(v)$. (For that matter, one can look at the distribution of is $(w)$ or ds $(w)$ where $w$ ranges over any "interesting" subset of $\mathfrak{S}_{n}$.) For instance, Reifegerste [83, Cor. 4.3] shows that for $k \geq 3$,

$$
\#\left\{w \in \mathfrak{S}_{n}(231): \text { is }(w)<k\right\}=\frac{1}{n} \sum_{i=1}^{k-1}\left(\begin{array}{c}
n \\
i
\end{array}\right)\left(\begin{array}{c}
n \\
i-1
\end{array}\right),
$$


a sum of Narayana numbers [99, Exer. 6.36]. Note that the left-hand side of (30) can also be written as $\#\left\{w \in \mathfrak{S}_{n}(231,12 \ldots k)\right\}$, the number of permutations in $\mathfrak{S}_{n}$ avoiding both 231 and $12 \ldots k$. Asymptotic results were obtained by Deutsch, Hildebrand, and Wilf [41] for the distribution of is $(w)$ when $v=231,132$, and 321. Their result for $v=132$ is the following.

Theorem 12. For $w \in \mathfrak{S}_{n}(132)$ the random variable is $(w)$ has mean $\sqrt{\pi n}+O\left(n^{1 / 4}\right)$ and standard deviation $\sqrt{\pi\left(\frac{\pi}{3}-1\right)} \sqrt{n}+O\left(n^{1 / 4}\right)$. Moreover, for any $t>-\sqrt{\pi}$ we have

$$
\lim _{n \rightarrow \infty} \operatorname{Prob}\left(\frac{\text { is }(w)-\sqrt{\pi n}}{\sqrt{n}} \leq t\right)=\sum_{j \in \mathbb{Z}}\left(1-2 j^{2}(t+\sqrt{\pi})^{2}\right) e^{-(t+\sqrt{\pi})^{2} j^{2}} .
$$

The proof of Theorem 12 is considerably easier than its counterpart for $w \in \mathfrak{S}_{n}$ (Theorem 4) because there is a relatively simple formula for the number $f(n, k)$ of permutations $w \in \mathfrak{S}_{n}(132)$ satisfying is $(w)<k$, viz.,

$$
f(n, k)=2 \sum_{i=\lceil-n /(k+1)\rceil}^{\lfloor(n+1) /(k+1)\rfloor}\left(\left(\begin{array}{c}
2 n \\
n+i(k+1)
\end{array}\right)-\frac{1}{4}\left(\begin{array}{c}
2 n+2 \\
n+1+i(k+1)
\end{array}\right)\right) .
$$

A number of variations and generalizations of pattern-avoiding permutations have been investigated. In particular, we can look at patterns where some of the terms must appear consecutively. This concept was introduced by Babson and Steingrímsson [10] and further investigated by Claesson [39] and others. For instance, the generalized pattern 1-32 indicates a subsequence $a_{i} a_{j} a_{j+1}$ of a permutation $w=a_{1} a_{2} \ldots a_{n}$ such that $a_{i}<a_{j+1}<a_{j}$. The hyphen in the notation 1-32 means that the first two terms of the subsequence need not be consecutive. The permutations in $\mathfrak{S}_{4}$ avoiding 1-32 are all $C_{4}=14$ permutations avoiding 132 (in the previous sense, so avoiding 1-3-2 in the present context) together with 2413. A typical result, due to Claesson [39, Props. 2 and 5], asserts that

$$
\# \mathfrak{S}_{n}(1-23)=\# \mathfrak{S}_{n}(1-32)=B(n),
$$

the number of partitions of the set $[n]$ (a Bell number $[98, \S 1.4])$.

\section{Unimodal and alternating subsequences}

We briefly discuss two variations of increasing/decreasing subsequences of a different flavor from those considered above. There is considerable room for further work in this area.

Early work of Chung [37] and Steele [102] deals with $k$-unimodal subsequences. A sequence is $k$-unimodal if it is a concatenation of (at most) $k+1$ monotone sequences. (In more traditional terminology, a sequence is $k$-unimodal if it has at most $k+1$ 
alternating runs $[30, \S 1.2]$.) Thus a 0-unimodal sequence is just an increasing or decreasing sequence, and every such sequence is $k$-unimodal for all $k$. The sequences 41235 and 24531 are 1-unimodal. Chung showed that every $w \in \mathfrak{S}_{n}$ has a 1-unimodal subsequence of length $\left[\sqrt{2 n+\frac{1}{4}}-\frac{1}{2}\right]$, and that this result is best possible. She conjectured that if $E_{k}(n)$ is the expected length of the longest $k$-unimodal subsequence of a random permutation $w \in \mathfrak{S}_{n}$, then $E_{k}(n) / \sqrt{n}$ approaches a limit $c_{k}$ as $n \rightarrow \infty$. Steele proved this conjecture and showed that $c_{k}=2 \sqrt{k+1}$ by deducing it from the monotone $(k=0)$ case.

A sequence $b_{1} b_{2} \ldots b_{k}$ of integers is alternating if

$$
b_{1}>b_{2}<b_{3}>b_{4}<\cdots b_{k} \text {. }
$$

For instance, there are five alternating permutations in $\mathfrak{S}_{4}$, viz., 2143, 3142, 4132, 3241,4231 . If $E_{n}$ denotes the number of alternating permutations in $\mathfrak{S}_{n}$, then a famous result of André [6], [99, §3.16] states that

$$
\sum_{n \geq 0} E_{n} \frac{x^{n}}{n !}=\sec x+\tan x
$$

The numbers $E_{n}$ were first considered by Euler (using (31) as their definition) and are known as Euler numbers. Sometimes $E_{2 n}$ is called a secant number and $E_{2 n-1}$ a tangent number.

We can try to extend the main results on increasing/decreasing subsequences to alternating subsequences. In particular, given $w \in \mathfrak{S}_{n}$ let as $(w)=\operatorname{as}_{n}(w)$ denote the length of the longest alternating subsequence of $w$, and define

$$
b_{k}(n)=\#\left\{w \in \mathfrak{S}_{n}: \text { as }(w) \leq k\right\} .
$$

Thus $b_{1}(n)=1$ (corresponding to the permutation $12 \ldots n$ ), $b_{k}(n)=n$ ! if $k \geq n$, and $b_{n}(n)-b_{n-1}(n)=E_{n}$. Note that we can also define $b_{k}(n)$ in terms of pattern avoidance, viz., $b_{k}(n)$ is the number of $w \in \mathfrak{S}_{n}$ avoiding all $E_{k+1}$ alternating permutations in $\mathfrak{S}_{k+1}$.

Unlike the situation for $u_{k}(n)$ (defined by (16)), there are "nice" explicit generating functions and formulas for $b_{k}(n)$. The basic reason for the existence of such explicit results is the following (easily proved) key lemma.

Lemma 13. For any $w \in \mathfrak{S}_{n}$, there exists an alternating subsequence of $w$ of maximum length that contains $n$.

From Lemma 13 it is straightforward to derive a recurrence satisfied by $a_{k}(n):=$ $b_{k}(n)-b_{k-1}(n)$, viz.,

$$
a_{k}(n)=\sum_{j=1}^{n}\left(\begin{array}{l}
n-1 \\
j-1
\end{array}\right) \sum_{2 r+s=k-1}\left(a_{2 r}(j-1)+a_{2 r+1}(j-1)\right) a_{s}(n-j) .
$$


Now define

$$
B(x, t)=\sum_{k, n \geq 0} b_{k}(n) t^{k} \frac{x^{n}}{n !} .
$$

It follows from the recurrence (32) (after some work [101]) that

$$
B(x, t)=\frac{1+\rho+2 t e^{\rho x}+(1-\rho) e^{2 \rho x}}{1+\rho-t^{2}+\left(1-\rho-t^{2}\right) e^{2 \rho x}},
$$

where $\rho=\sqrt{1-t^{2}}$. Alternatively (as pointed out by M. Bóna), let $G(n, k)$ denote the number of $w \in \mathfrak{S}_{n}$ with $k$ alternating runs as defined at the beginning of this section. Then equation (33) is a consequence of the relation $a_{k}(n)=\frac{1}{2}(G(n, k-1)+G(n, k))$ and known facts about $G(n, k)$ summarized in [30, §1.2].

It can be deduced from equation (33) (shown with assistance from I. Gessel) that

$$
b_{k}(n)=\frac{1}{2^{k-1}} \sum_{\substack{i+2 j \leq k \\
i \equiv k(\bmod 2)}}(-2)^{j}\left(\begin{array}{c}
k-j \\
(k+i) / 2
\end{array}\right)\left(\begin{array}{l}
n \\
j
\end{array}\right) i^{n} .
$$

For instance,

$b_{1}(n)=1, \quad b_{2}(n)=2^{n-1}, \quad b_{3}(n)=\frac{1}{4}\left(3^{n}-2 n+3\right), \quad b_{4}(n)=\frac{1}{8}\left(4^{n}-(2 n-4) 2^{n}\right)$.

From equation (33) it is also easy to compute the moments

$$
M_{k}(n)=\frac{1}{n !} \sum_{w \in \mathfrak{S}_{n}} \operatorname{as}_{n}(w)^{k}
$$

For instance,

$$
\sum_{n \geq 1} M_{1}(n) x^{n}=\left.\frac{\partial B(x, t)}{\partial t}\right|_{t=1}=\frac{6 x-3 x^{2}+x^{3}}{6(1-x)^{2}},
$$

from which we obtain

$$
M_{1}(n)= \begin{cases}1, & n=1 \\ \frac{4 n+1}{6}, & n>1 .\end{cases}
$$

Similarly the variance of $\operatorname{as}_{n}(w)$ is given by

$$
\operatorname{Var}\left(\operatorname{as}_{n}\right)=\frac{8}{45} n-\frac{13}{180}, \quad n \geq 4
$$

It is surprising that there are such simple explicit formulas, in contrast to the situation for is $(w)$ (equation (10)).

It is natural to ask for the limiting distribution of $\mathrm{as}_{n}$, analogous to Theorem 4 for is $s_{n}$. The following result was shown independently by R. Pemantle [79] and H. Widom [114]. It can also be obtained by showing that the polynomials $\sum_{k} a_{k}(n) t^{k}$ have (interlacing) real zeros, a consequence of the connection between $a_{k}(n)$ and $G(n, k)$ mentioned above and a result of Wilf [30, Thm. 1.41]. 
Theorem 14. We have for random (uniform) $w \in \mathfrak{S}_{n}$ and all $t \in \mathbb{R}$ that

$$
\lim _{n \rightarrow \infty} \operatorname{Prob}\left(\frac{\operatorname{as}_{n}(w)-2 n / 3}{\sqrt{n}} \leq t\right)=G(t)
$$

where $G(t)$ is Gaussian with variance 8/45:

$$
G(t)=\frac{1}{\sqrt{\pi}} \int_{-\infty}^{t \sqrt{45} / 4} e^{-s^{2}} d s .
$$

For further information on longest alternating subsequences, see the paper [101].

\section{Matchings}

The subject of pattern containment and avoidance discussed in Section 7 provides one means to extend the concept of increasing/decreasing subsequences of permutations. In this section we will consider a different approach, in which permutations are replaced with other combinatorial objects. We will be concerned mainly with (complete) matchings on [2n], which may be defined as partitions $M=\left\{B_{1}, \ldots, B_{n}\right\}$ of [ $2 n]$ into $n$ two-element blocks $B_{i}$. Thus $B_{1} \cup B_{2} \cup \cdots \cup B_{n}=[2 n], B_{i} \cap B_{j}=\emptyset$ if $i \neq j$, and $\# B_{i}=2$. (These conditions are not all independent.) Alternatively, we can regard a matching $M$ as a fixed-point free involution $w_{M}$ of [2n], viz., if $B_{i}=\{a, b\}$ then $w_{M}(a)=b$. We already considered increasing and decreasing subsequences of fixed-point free involutions in Section 5. In that situation, however, there is no symmetry interchanging increasing subsequences with decreasing subsequences. Here we consider two alternative statistics on matchings (one of which is equivalent to decreasing subsequences) which have the desired symmetry.

Write $\mathfrak{M}_{n}$ for the set of matchings on [2n]. We represent a matching $M \in \mathfrak{M}_{n}$ by a diagram of $2 n$ vertices $1,2, \ldots, 2 n$ on a horizontal line in the plane, with an arc between vertices $i$ and $j$ and lying above the vertices if $\{i, j\}$ is a block of $M$. Figure 4 shows the diagram corresponding to the matching

$$
M=\{\{1,5\},\{2,9\},\{3,10\},\{4,8\},\{6,7\}\} .
$$

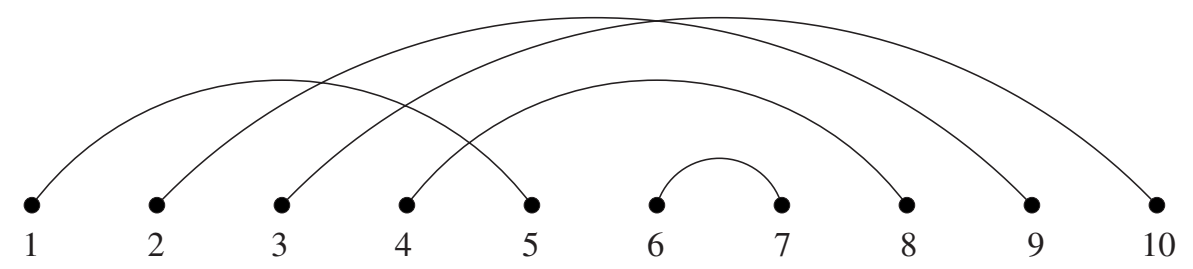

Figure 4. A matching on [10]. 
Let $M \in \mathfrak{M}_{n}$. A crossing of $M$ consists of two $\operatorname{arcs}\{i, j\}$ and $\{k, l\}$ with $i<$ $k<j<l$. Similarly a nesting of $M$ consists of two arcs $\{i, j\}$ and $\{k, l\}$ with $i<k<l<j$. The maximum number of mutually crossing arcs of $M$ is called the crossing number of $M$, denoted $\operatorname{cr}(M)$. Similarly the nesting number ne $(M)$ is the maximum number of mutually nesting arcs. For the matching $M$ of Figure 4, we have $\operatorname{cr}(M)=3$ (corresponding to the $\operatorname{arcs}\{1,5\},\{2,9\}$, and $\{3,10\}$ ), while also ne $(M)=3$ (corresponding to $\{2,9\},\{4,8\}$, and $\{6,7\}$ ).

It is easy to see that $\mathrm{ds}\left(w_{M}\right)=2 \cdot \operatorname{ne}(M)$, where $w_{M}$ is the fixed-point free involution corresponding to $M$ as defined above. However, it is not so clear whether $\operatorname{cr}(M)$ is connected with increasing/decreasing subsequences. To this end, define

$$
f_{n}(i, j)=\#\left\{M \in \mathfrak{M}_{n}: \operatorname{cr}(M)=i, \operatorname{ne}(M)=j\right\} .
$$

It is well-known that

$$
\sum_{j} f_{n}(0, j)=\sum_{i} f_{n}(i, 0)=C_{n}
$$

In other words, the number of matchings $M \in \mathfrak{M}_{n}$ with no crossings (or with no nestings) is the Catalan number $C_{n}$. For crossings this result goes back to Errera [44], [99, Exer. 6.19(n,o)]; for nestings see [100]. Equation (36) was given the following generalization by Chen et al. [36].

Theorem 15. For all $i, j, n$ we have $f_{n}(i, j)=f_{n}(j, i)$.

Theorem 15 is proved by using a version of RSK first defined by the author (unpublished) and then extended by Sundaram [103]. Define an oscillating tableau of shape $\lambda \vdash n$ and length $k$ to be a sequence

$$
\emptyset=\lambda^{0}, \lambda^{1}, \ldots, \lambda^{k}=\lambda
$$

of partitions $\lambda^{i}$ such that (the diagram of) $\lambda^{i+1}$ is obtained from $\lambda^{i}$ by either adding or removing a square. (Note that if we add a square each time, so $k=n$, then we obtain an SYT of shape $\lambda$.) Oscillating tableaux were first defined (though not with that name) by Berele [22] in connection with the representation theory of the symplectic group. Given a matching $M \in \mathfrak{M}_{n}$, define an oscillating tableau $\Phi(M)=\left(\lambda^{0}, \lambda^{1}, \ldots, \lambda^{2 n}\right)$ of length $2 n$ and shape $\emptyset$ as follows. Label the right-hand endpoints of the $\operatorname{arcs}$ of $M$ by $1,2, \ldots, n$ from right-to-left. Label each left-hand endpoint with the same label as the right-hand endpoint. Begin with the empty tableau $T_{0}=\emptyset$. Let $a_{1}, \ldots, a_{2 n}$ be the sequence of labels, from left-to-right. Once $T_{i-1}$ has been obtained, define $T_{i}$ to be the tableau obtained by row-inserting $a_{i}$ into $T_{i-1}$ (as defined in Section 2) if $a_{i}$ is the label of a left-hand endpoint of an arc; otherwise $T_{i}$ is the tableau obtained by deleting $a_{i}$ from $T_{i-1}$. Let $\lambda^{i}$ be the shape of $T_{i}$, and set

$$
\Phi(M)=\left(\emptyset=\lambda^{0}, \lambda^{1}, \ldots, \lambda^{2 n}=\emptyset\right) .
$$


See Figure 5 for an example. It is easy to see that $\Phi(M)$ is an oscillating tableau of length $2 n$ and shape $\emptyset$. With a little more work it can be shown that in fact the map $M \mapsto \Phi(M)$ is a bijection from $\mathfrak{M}_{n}$ to the set $\mathcal{O}_{n}$ of all oscillating tableaux of length $2 n$ and shape $\emptyset$. As a consequence we have the enumerative formula

$$
\# \mathcal{O}_{n}=(2 n-1) ! !:=1 \cdot 3 \cdot 5 \ldots(2 n-1),
$$

the number of matchings on $[2 n]$. The key fact about the correspondence $\Phi$ for proving Theorem 15 is the following "oscillating analogue" of Schensted's theorem (Theorem 2).

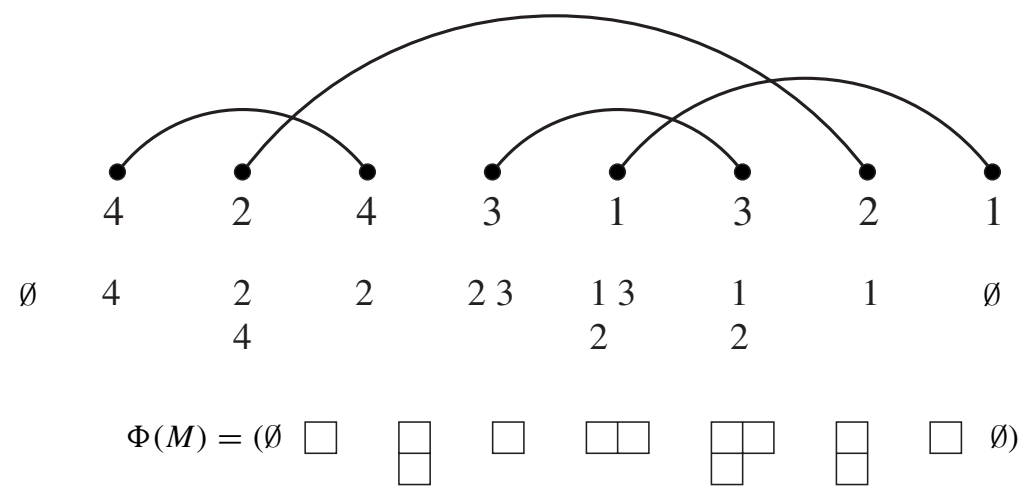

Figure 5. A correspondence between matchings and oscillating tableaux.

Theorem 16. Let $M \in \mathfrak{M}_{n}$, and suppose that $\Phi(M)=\left(\lambda^{0}, \lambda^{1}, \ldots, \lambda^{2 n}\right)$. Then $\mathrm{ne}(M)$ is equal to the most number of columns of any $\lambda^{i}$, while $\operatorname{cr}(M)$ is equal to the most number of rows of any $\lambda^{i}$.

Theorem 15 is an easy consequence of Theorem 16. For let $\Phi(M)^{\prime}$ be the oscillating tableau obtained by conjugating all the partitions in $\Phi(M)$, and let $M^{\prime}$ be the matching satisfying $\Phi\left(M^{\prime}\right)=\Phi(M)^{\prime}$. By Theorem 16 we have $\operatorname{cr}(M)=\operatorname{ne}\left(M^{\prime}\right)$ and ne $(M)=\operatorname{cr}\left(M^{\prime}\right)$. Since the map $M \mapsto \Phi(M)$ is a bijection we have that the operation $M \mapsto M^{\prime}$ is a bijection from $\mathfrak{M}_{n}$ to itself that interchanges cr and ne, and the proof follows.

The above argument and equation (7) show that the operation $M \mapsto M^{\prime}$ on matchings is a natural analogue of the operation of reversal on permutations. Unlike the case of permutations, we do not know a simple "direct" operation on matchings that interchanges cr with ne.

Let $\tilde{f}_{n}^{\lambda}$ denote the number of oscillating tableaux of shape $\lambda$ and length $n$, so $\tilde{f}_{n}^{\lambda}=0$ unless $n \equiv|\lambda|(\bmod 2)$. A generalization for $\tilde{f}_{n}^{\lambda}$ of the hook-length formula (equation (3)) is due to Sundaram [103, Lemma 2.2], viz.,

$$
\tilde{f}_{n}^{\lambda}=\left(\begin{array}{l}
n \\
k
\end{array}\right)(n-k-1) ! ! f^{\lambda}
$$


where $\lambda \vdash k$ and where of course $f^{\lambda}$ is evaluated by the usual hook-length formula (3). (Set $(-1) ! !=1$ when $n=k$.) Note that an oscillating tableau $\left(\lambda^{0}, \lambda^{1}, \ldots, \lambda^{2 n}\right)$ of shape $\emptyset$ may be regarded as a pair $(P, Q)$ of oscillating tableaux of the same shape $\lambda=\lambda^{n}$ and length $n$, viz.,

$$
\begin{aligned}
& P=\left(\lambda^{0}, \lambda^{1}, \ldots, \lambda^{n}\right), \\
& Q=\left(\lambda^{2 n}, \lambda^{2 n-1}, \ldots, \lambda^{n}\right) .
\end{aligned}
$$

Hence we obtain the following restatement of equation (37):

$$
\sum_{\lambda}\left(\tilde{f}_{n}^{\lambda}\right)^{2}=(2 n-1) ! !
$$

where $\lambda$ ranges over all partitions. (The partitions $\lambda$ indexing a nonzero summand are those satisfying $\lambda \vdash k \leq n$ and $k \equiv n(\bmod 2)$.)

Equation (38) suggests, in analogy to equations (1) and (2), a connection between $\tilde{f}_{n}^{\lambda}$ and representation theory. Indeed, there is a $\mathbb{C}$-algebra $\mathfrak{B}_{n}(x)$, where $x$ is a real parameter, which is semisimple for all but finitely many $x$ (and such that these exceptional $x$ are all integers) and which has a basis that is indexed in a natural way by matchings $M \in \mathfrak{M}_{n}$. In particular, $\operatorname{dim} \mathfrak{B}_{n}(x)=(2 n-1)$ !!. This algebra was first defined by Brauer [32] and shown to be the centralizer algebra of the action of the orthogonal group $\mathrm{O}(V)$ on $V^{\otimes n}$ (the $n$th tensor power of $V$ ), where $\operatorname{dim} V=k$ and $x=k$. It is also the centralizer algebra of the action of the symplectic group $\operatorname{Sp}(2 k)$ on $V^{\otimes n}$, where now $\operatorname{dim} V=2 k$ and $x=-2 k$. When $\mathfrak{B}_{n}(x)$ is semisimple, its irreducible representations have dimension $\tilde{f}_{n}^{\lambda}$, so we obtain a representation-theoretic explanation of equation (38). For further information see e.g. Barcelo and Ram [21, App. B6]

Because ds $\left(w_{M}\right)=2 \cdot \operatorname{ne}(M)$ and because $\mathrm{cr}_{n}$ and $\mathrm{ne}_{n}$ have the same distribution by Theorem 15, the asymptotic distribution of $\mathrm{cr}_{n}$ and $\mathrm{ne}_{n}$ on $\mathfrak{M}_{n}$ reduces to that of $\mathrm{ds}_{2 n}$ on $\mathfrak{I}_{2 n}^{*}$, which is given by Theorem $9(\mathrm{~b})$. We therefore obtain the following result.

Theorem 17. We have for random (uniform) $M \in \mathfrak{M}_{n}$ and all $t \in \mathbb{R}$ that

$$
\lim _{n \rightarrow \infty} \operatorname{Prob}\left(\frac{\mathrm{ne}_{n}(M)-\sqrt{2 n}}{(2 n)^{1 / 6}} \leq \frac{t}{2}\right)=F(t)^{1 / 2} \exp \left(\frac{1}{2} \int_{t}^{\infty} u(s) d s\right) .
$$

The same result holds with $\mathrm{ne}_{n}$ replaced with $\mathrm{cr}_{n}$.

We can also consider the effect of bounding both $\operatorname{cr}(M)$ and ne $(M)$ as $n \rightarrow \infty$. The analogous problem for is $(w)$ and $\mathrm{ds}(w)$ is not interesting, since by Theorem 1 there are no permutations $w \in \mathfrak{S}_{n}$ satisfying is $(w) \leq p$ and ds $(w) \leq q$ as soon as $n>p q$. Let

$$
\begin{aligned}
& h_{p, q}(n)=\#\left\{M \in \mathfrak{M}_{n}: \operatorname{cr}(M) \leq p, \operatorname{ne}(M) \leq q\right\} \\
& H_{p, q}(x)=\sum_{n \geq 0} h_{p, q}(n) x^{n}
\end{aligned}
$$


It follows from the bijection $\Phi: \mathfrak{M}_{n} \rightarrow \mathcal{O}_{n}$ and a simple application of the transfermatrix method $[98, \S 4.7]$ that $H_{p, q}(x)$ is a rational function of $x[36, \S 5]$. For instance,

$$
\begin{gathered}
H_{1,1}(x)=\frac{1}{1-x}, \quad H_{1,2}(x)=\frac{1-x}{1-2 x}, \quad H_{1,3}(x)=\frac{1-2 x}{1-3 x+x^{2}} \\
H_{2,2}(x)=\frac{1-5 x+2 x^{2}}{(1-x)(1-5 x)}, \quad H_{2,3}(x)=\frac{1-11 x+30 x^{2}-23 x^{3}+4 x^{4}}{(1-x)(1-3 x)\left(1-8 x+4 x^{2}\right)} \\
H_{3,3}(x)=\frac{1-24 x+186 x^{2}-567 x^{3}+690 x^{4}-285 x^{5}+15 x^{6}}{(1-x)\left(1-19 x+83 x^{2}-x^{3}\right)\left(1-5 x+6 x^{2}-x^{3}\right)^{2}} .
\end{gathered}
$$

Christian Krattenthaler pointed out that $h_{p, q}(n)$ can be interpreted as counting certain walks in an alcove of the affine Weyl group $\tilde{C}_{n}$. It then follows from a result of Grabiner $[49,(23)]$ that all reciprocal zeros of the denominator of $H_{p, q}(x)$ are of the form

$$
2\left(\cos \left(\pi r_{1} / m\right)+\cdots+\cos \left(\pi r_{j} / m\right)\right)
$$

where each $r_{i} \in \mathbb{Z}$ and $m=p+q+1$. All these numbers for fixed $m$ belong to an extension of $\mathbb{Q}$ of degree $\phi(2 m) / 2$, where $\phi$ is the Euler phi-function. As a consequence, every irreducible factor (over $\mathbb{Q}$ ) of the denominator of $H_{p, q}(x)$ has degree dividing $\phi(2 m) / 2$.

Theorem 15 can be extended to objects other than matchings, in particular, arbitrary set partitions. (Recall that we have defined a matching to be a partition of [2n] into $n$ 2-element blocks.) In this situation oscillating tableaux are replaced by certain sequences of partitions known as vacillating tableaux. See [36] for further details. Vacillating tableaux were introduced implicitly (e.g., [54, (2.23)]) in connection with the representation theory of the partition algebra $\mathfrak{P}_{n}$, a semisimple algebra whose dimension is the Bell number $B(n)$. See Halverson and Ram [54] for a survey of the partition algebra. Vacillating tableaux and their combinatorial properties were made more explicit by Chen, et al. [36] and by Halverson and Lewandowski [53]. An alternative approach based on "growth diagrams" to vacillating tableaux and their nesting and matchings was given by Krattenthaler [68]. It remains open to find an analogue of Theorem 17 for the distribution of $\operatorname{cr}(\pi)$ or ne $(\pi)$ (as defined in [36]) for arbitrary set partitions $\pi$.

\section{References}

[1] Albert, M. H., On the length of the longest subsequence avoiding an arbitrary pattern in a random permutation. Technical Report OUCS-2005-08, University of Otago, 2005; math.CO/0505485.

[2] Albert, M. H., Aldred, R. E. L., Atkinson, M. D., Handley, C. C., Holton, D. A., McCaughan, D. J., Sagan, B. E., Monotonic sequence games. In Games of No Chance III, submitted; math.CO/0602630. 
[3] Albert, M. H., Elder, M., Rechnitzer, A., Westcott, P., Zabrocki, M., On the Stanley-Wilf limit of 4231-avoiding permutations and a conjecture of Arratia. Adv. in Appl. Math. 36 (2) (2006), 96-105.

[4] Aldous, D. J., Diaconis, P., Hammersley's interacting particle process and longest increasing subsequences. Probab. Theory Related Fields 103 (1995), 199-213.

[5] Aldous D. J., Diaconis, P., Longest increasing subsequences, from patience sorting to the Baik-Deift-Johansson theorem. Bull. Amer. Math. Soc. 36 (1999), 413-432.

[6] André, D., Développement de $\sec x$ and $\operatorname{tg} x$. C. R. Math. Acad. Sci. Paris 88 (1879), 965-979.

[7] Andrews, G. E., Askey, R., Roy, R., Special Functions. Encyclopedia of Mathematics and Its Applications 71, Cambridge University Press, Cambridge, New York 1999.

[8] Arratia, A. On the Stanley-Wilf conjecture for the number of permutations avoiding a given pattern. Electron. J. Combin. 6 (1) (1999), Article N1 (electronic).

[9] Atkinson, M., Holton, D. (eds.), Special volume on permutation patterns. Electron. J. Combin. 9 (2002-2003) (electronic).

[10] Babson, E., Steingrímsson, E., Generalized permutation patterns and a classification of the Mahonian statistics. Sém. Lothar. Combin. 44 (2000), Article B44b (electronic).

[11] Bachmat, E., Analysis of disk scheduling with linear seek function, increasing, subsequences, and space-time geometry. Preprint; math.OC/0601025.

[12] Bachmat, E., Berend, D., Sapir, L., Skiena, S., Stolyarov, N., Analysis of airplane boarding via space-time geometry and random matrix theory. Preprint; physics/0512020.

[13] Bachmat, E., Berend, D., Sapir, L., Skiena, S., Stolyarov, N., Analysis of airplane boarding times. Preprint.

[14] Baer, R. M., Brock, P., Natural sorting over permutation spaces. Math. Comp. 22 (1968), 385-410.

[15] Baik, J., Deift, P., Johansson, K., On the distribution of the length of the longest increasing subsequence of random permutations. J. Amer. Math. Soc. 12 (1999), 1119-1178.

[16] Baik, J., Deift, P., Johansson, K., On the distribution of the length of the second row of a Young diagram under the Plancherel measure. Geom. Funct. Anal. 10 (2000), 702-731; Addendum, ibid. 1606-1607.

[17] Baik, J., Rains, E., Algebraic aspects of increasing subsequences. Duke Math. J. 109 (2001), 1-65.

[18] Baik, J., Rains, E., Symmetrized random permutations. In Random Matrix Models and Their Applications, Math. Sci. Res. Inst. Publ. 40, Cambridge University Press, Cambridge 2001, 1-19.

[19] Baik, J., Rains, E., The asymptotics of monotone subsequences of involutions. Duke Math. J. 109 (2001), 205-281.

[20] Banderier, C., Bousquet-Mélou, M., Denise, A., Flajolet, P., Gardy, D., GouyouBeauchamps, D., Generating functions for generating trees. Discrete Math. 246 (2002), 29-55.

[21] Barcelo, H., Ram, A., Combinatorial representation theory. In New Perspectives in Algebraic Combinatorics (Berkeley, CA, 1996-97), Math. Sci. Res. Inst. Publ. 38, Cambridge University Press, Cambridge 1999, 23-90. 
[22] Berele, A., A Schensted-type correspondence for the symplectic group. J. Combin. Theory Ser. A 43 (1986), 320-328.

[23] Bergeron, F., Favreau, L., Krob, D., Conjectures on the enumeration of tableaux of bounded height. Discrete Math. 139 (1995), 463-468.

[24] Bergeron, F., Gascon, F., Counting Young tableaux of bounded height. J. Integer Seq. 3 (2000), Article 00.1.7 (electronic).

[25] Billey, S. C., Jockusch, W., Stanley, R., Some combinatorial properties of Schubert polynomials. J. Algebraic Combin. 2 (1993), 345-374.

[26] Billey, S. C., Lakshmibai, V., Singular Loci of Schubert Varieties. Progr. Math. 182, Birkäuser, Boston, MA, 2000.

[27] Billey, S. C., Warrington, G. S., Kazhdan-Lusztig polynomials for 321-hexagon-avoiding permutations. J. Algebraic Combin. 13 (2001), 111-136.

[28] Bóna, M., Exact enumeration of 1342-avoiding permutations: a close link with labeled trees and planar maps. J. Combin. Theory Ser. A 80 (1997), 257-272.

[29] Bóna, M., The permutation classes equinumerous to the smooth class. Electron. J. Combin. 5 (1998), Article R31 (electronic).

[30] Bóna, M., Combinatorics of Permutations. Chapman \& Hall/CRC, Boca Raton, FL, 2004.

[31] Borodin, A., Okounkov, A. Olshanski, G., Asymptotics of Plancherel measures for symmetric groups. J. Amer. Math. Soc. 13 (2000), 481-515.

[32] Brauer, R., On algebras which are connected with the semisimple continuous groups. Ann. Math. 38 (1937), 854-872.

[33] Burstein, A., Lankham, I., Combinatorics of patience sorting piles. In Proc. Formal Power Series and Algebraic Combinatorics (Taormina, Sicily, 2005), Sém. Lothar. Combin. 54A (2005/06), electronic.

[34] Burstein, A., Lankham, I., A geometric form for the extended patience sorting algorithm. Adv. in Appl. Math. 36 (2) (2006), 106-117.

[35] Burstein, A., and Lankham, I., Restricted patience sorting and barred pattern avoidance. In Proc. Formal Power Series and Algebraic Combinatorics (June 2006), to appear; math.CO/0512122.

[36] Chen, W. Y. C., Deng, E. Y. P., Du, R. R. X., Stanley, R., Yan, C. H., Crossings and nestings of matchings and partitions. Trans. Amer. Math. Soc., to appear; math.CO/0501230.

[37] Chung, F., On unimodal subsequences. J. Combin. Theory Ser. A 29 (1980), 267-279.

[38] Chung, F. R. K., Graham, R. L., Hoggatt, V. E., Kleiman, M., The number of Baxter permutations. J. Combin. Theory Ser. A 24 (1978), 382-394.

[39] Claesson, A., Generalized pattern avoidance. European J. Combin. 22 (2001), 961-971.

[40] Deift, P., Integrable systems and combinatorial theory. Notices Amer. Math. Soc. 47 (2000), 631-640.

[41] Deutsch, E., Hildebrand, A. J., Wilf, H., The distribution of longest increasing subsequences in pattern-restricted permutations. Electron. J. Combin. 9(2) (2002-2003), Article R12 (electronic).

[42] Diaconis, P., Shahshahani, M., On the eigenvalues of random matrices. J. Appl. Prob. 31 (1994), 49-61. 
[43] Erdős, P., Szekeres, G., A combinatorial problem in geometry. Composito Math. 2 (1935), 463-470.

[44] Errera, A., Analysis situs. Un problème d'énumeration. Mém. Acad. Roy. Belgique Coll. $8^{\circ}(2) 11$ (1931), $26 \mathrm{pp}$.

[45] Frame, J. S., Robinson, G. de B., Thrall, R. M., The hook graphs of $S_{n}$. Canad. J. Math. 6 (1954), 316-324.

[46] Gardner, M., The Last Recreations. Springer-Verlag, New York 1997; reprinted from Scientific American 216 (March 1967), 124-129, and 216 (April 1967), 116-123.

[47] Gessel, I. M., Symmetric functions and P-recursiveness. J. Combin. Theory Ser. A 53 (1990), 257-285.

[48] Goulden, I. P., A linear operator for symmetric functions and tableaux in a strip with given trace. Discrete Math. 99 (1992), 69-77.

[49] Grabiner, D., Random walk in an alcove of an affine Weyl group, and non-colliding random walks on an interval. J. Combin. Theory Ser. A 97 (2002), 285-306.

[50] Greene, C., An extension of Schensted's theorem. Adv. Math. 14 (1974), 254-265.

[51] Groeneboom, P., Ulam's problem and Hammersley's process. Ann. Probab. 29 (2001), 683-690.

[52] Haiman, M., unpublished.

[53] Halverson, T., Lewandowski, T., RSK insertion for set partitions and diagram algebras. Electron. J. Combin. 11 (2) (2004-2005), Article R24 (electronic).

[54] Halverson, T., Ram, A., Partition algebras. European J. Combin. 26 (2005), 869-921.

[55] Harary, F., Sagan, B., West, D, Computer-aided analysis of monotonic sequence games. Atti Accad. Peloritana Cl. Sci. Fis. Mat. Natur. 61 (1983), 67-78.

[56] Hammersley, J. M., A few seedlings of research. In Proc. Sixth Berkeley Symposium on Mathematical Statistics and Probability (Berkeley, 1970/1971), Vol. 1: Theory of statistics. University California Press, Berkeley, CA, 1972, 345-394.

[57] Ivanov, V., Olshanski, G., Kerov's central limit theorem for the Plancherel measure on Young diagrams. In Symmetric Functions 2001: Surveys of Developments and Perspectives (S. Fomin, ed.), NATO Sci. Ser. II Math. Phys. Chem. 74, Kluwer, Dordrecht 2002, 93-151.

[58] Johansson, K., The longest increasing subsequence in a random permutation and a unitary random matrix model. Math. Res. Lett. 5 (1998), 63-82.

[59] Johansson, K., Discrete orthogonal polynomial ensembles and the Plancherel measure. Ann. Math. 153 (2001), 259-296.

[60] Johansson, K., Toeplitz determinants, random growth and determinantal processes. In Proceedings of the International Congress of Mathematicians (Beijing, 2002), Vol. III, Higher Ed. Press, Beijing 2002, 53-62.

[61] Kerov, S. V., Gaussian limit for the Plancherel measure of the symmetric group. $C$. $R$. Acad. Sci. Paris Sér. I Math. 316 (1993), 303-308.

[62] Kerov, S. V., Asymptotics of the separation of roots of orthogonal polynomials. Algebra i Analiz 5 (1993), 68-86; English translation: St. Petersburg Math. J. 5 (1994), 925-941. 
[63] Kerov, S. V., The asymptotics of interlacing sequences and the growth of continual Young diagrams. Zap. Nauchn. Sem. S.-Peterburg. Otdel. Mat. Inst. Steklov. (POMI) 205 (1993), Differentsialnaya Geom. Gruppy Li i Mekh. 13, 21-29, 179; English translation: J. Math. Sci. 80 (1996), 1760-1767.

[64] Kerov, S. V., Asymptotic Representation Theory of the Symmetric Group and Its Applications in Analysis. Transl. Math. Monogr. 219, Amer. Math. Soc., Providence, RI, 2003.

[65] Knuth, D. E., Permutations, matrices, and generalized Young tableaux. Pacific J. Math. 34 (1970), 709-727.

[66] Knuth, D. E., The Art of Computer Programming, Vol. 1, Fundamental Algorithms. Addison-Wesley, Reading, MA, 1968; second edition, 1973.

[67] Knuth, D. E., The Art of Computer Programming, Vol. 3, Sorting and Searching. AddisonWesley, Reading, MA, 1973; second edition, 1998.

[68] Krattenthaler, C., Growth diagrams, and increasing and decreasing chains in fillings of Ferrers shapes. Adv. in Appl. Math. 37 (2006), 404-431.

[69] Lakshmibai, V., Sandhya, B., Criterion for smoothness of Schubert varieties in $\mathrm{Sl}(n) / B$. Proc. Indian Acad. Sci. (Math. Sci.) 100 (1990), 45-52.

[70] van Leeuwen, M. A. A., The Robinson-Schensted and Schützenberger algorithms, an elementary approach. Electron. J. Combin. 3 (2) (1996), Article R15 (electronic).

[71] Logan, B. F., Shepp, L. A., A variational problem for random Young tableaux. Adv. Math. 26 (1977), 206-222.

[72] Macdonald, I. G., Symmetric Functions and Hall Polynomials. Second ed., Oxford Mathematical Monographs, The Clarendon Press, Oxford University Press, Oxford 1995.

[73] Macdonald, I. G., Notes on Schubert Polynomials. Publ. Laboratoire de Combinatoire et d'Informatique Mathématique 6, Université du Québec à Montréal, 1991.

[74] MacMahon, P. M., Memoir on the theory of the partitions of numbers - Part I. Phil. Trans. Royal Soc. London Ser. A 187 (1897), 619-673; Collected Works, Vol. 1 (G. E. Andrews, ed.), M.I.T. Press, Cambridge, MA, 1978, 1026-1080.

[75] Manivel, L., Symmetric functions, Schubert polynomials and degeneracy loci. SMF/AMS Texts and Monographs 6, Amer. Math. Soc., Providence, RI, and Soc. Math. France, Paris 2001.

[76] Marcus, A., Tardos, G., Excluded permutation matrices and the Stanley-Wilf conjecture. J. Combin. Theory Ser. A 107 (2004), 153-160.

[77] Morris, W., Soltan, V., The Erdős-Szekeres problem on points in convex position-a survey. Bull. Amer. Math. Soc. (N.S.) 37 (2000), 437-458.

[78] Okounkov, A., Random matrices and random permutations. Internat. Math. Res. Notices 2000 (2000), 1043-1095.

[79] Pemantle, R., private communications dated October 7 and October 9, 2005.

[80] Pittel, B., Romik, D., Limit shapes for random square Young tableaux and plane partitions. Preprint; math.PR/0505190.

[81] Rains, E., Increasing subsequences and the classical groups. Electron. J. Combin. 5 (1998), Article R12 (electronic).

[82] Regev, A., Asymptotic values for degrees associated with strips of Young diagrams. Adv. Math. 41 (1981), 115-136. 
[83] Reifegerste, A., On the diagram of 132-avoiding permutations. European J. Combin. 24 (2003), 759-776 (electronic).

[84] Robinson, G. de B., On the representations of $S_{n}$. Amer. J. Math. 60 (1938), 745-760.

[85] Romik, D., Permutations with short monotone subsequences. Adv. Appl. Math., to appear; http://www.stat.berkeley.edu/ romik/papers.html.

[86] Rotem, D., On a correspondence between binary trees and a certain type of permutation. Information Processing Lett. 4 (1975/76), 58-61.

[87] Ryan, K. M., On Schubert varieties in the flag manifold of $\mathrm{Sl}(n, C)$. Math. Ann. 276 (1987), 205-224.

[88] Sagan, B., The Symmetric Group. Second ed., Grad. Texts in Math. 203, Springer-Verlag, New York 2001.

[89] Schensted, C. E., Longest increasing and decreasing subsequences. Canad. J. Math. 13 (1961), 179-191.

[90] Schützenberger, M. P., Quelques remarques sur une construction de Schensted. Math. Scand. 12 (1963), 117-128.

[91] Schützenberger, M. P., Promotion des morphismes d'ensembles ordonnés. Discrete Math. 2 (1972), 73-94

[92] Seidenberg, A., A simple proof of a theorem of Erdős and Szekeres. J. London Math. Soc. 34 (1959), 352.

[93] Seppäläinen, T., Large deviations for increasing sequences on the plane. Probab. Theory Related Fields 112 (1998), 221-244.

[94] Simion, R., Schmidt, F., Restricted permutations. European J. Combin. 6 (1985), 383-406.

[95] Stankova, Z., West, J., Explicit enumeration of 321, hexagon-avoiding permutations. Discrete Math. 280 (2004), 165-189.

[96] Stanley, R., Differentiably finite power series. European J. Combin. 1 (1980), 175-188.

[97] Stanley, R., On the number of reduced decompositions of elements of Coxeter groups. European J. Combin. 5 (1984), 359-372.

[98] Stanley, R., Enumerative Combinatorics. Vol. 1, Wadsworth and Brooks/Cole, Pacific Grove, CA, 1986; second printing, Cambridge University Press, New York, Cambridge 1996.

[99] Stanley, R., Enumerative Combinatorics. Vol. 2. Cambridge University Press, New York, Cambridge 1999.

[100] Stanley, R., Catalan Addendum; http://www-math.mit.edu/ rstan/ec/catadd.pdf.

[101] Stanley, R., Longest alternating subsequences of permutations. Preprint; math.CO/ 0511419.

[102] Steele, M. J., Long unimodal subsequences: a problem of F. R. K. Chung. Discrete Math. 33 (1981), 223-225.

[103] Sundaram, S., The Cauchy identity for $\operatorname{Sp}(2 n)$. J. Combin. Theory Ser. A 53 (1990), 209-238.

[104] Tenner, B. E., Pattern avoidance and the Bruhat order. J. Combin. Theory Ser. A, to appear.

[105] Tenner, B. E., Database of permutation pattern avoidance. http://www-math.mit.edu/ $\sim$ bridget/cgi-bin/dppa.cgi. 
[106] Tracy, C. A., Widom, H., Level-spacing distributions and the Airy kernel. Comm. Math. Phys. 159 (1994), 151-174.

[107] Tracy, C. A., Widom, H., On orthogonal and symplectic ensembles. Comm. Math. Phys. 177 (1996), 727-754.

[108] Tracy, C. A., Widom, H., Distribution functions for largest eigenvalues and their applications. In Proc. International Congress of Mathematicians (Beijing 2002), Vol. I, Higher Ed. Press, Beijing 2002, 587-596.

[109] Ulam, S. M., Monte Carlo calculations in problems of mathematical physics. In Modern Mathematics for the Engineer: Second Series (ed. by E. F. Beckenbach), McGraw-Hill, New York 1961, 261-281.

[110] Vershik, A. M., Kerov, S. V., Asymptotic behavior of the Plancherel measure of the symmetric group and the limit form of Young tableaux . Dokl. Akad. Nauk SSSR 223 (1977), 1024-1027; English translation: Soviet Math. Dokl. 233 (1977), 527-531.

[111] West, J., Permutations with forbidden subsequences; and, stack-sortable permutation. Ph.D. thesis, M.I.T., 1990.

[112] West, J., Generating trees and the Catalan and Schröder numbers. Discrete Math. 146 (1995), 247-262.

[113] West, J., Generating trees and forbidden subsequences. Discrete Math. 157 (1996), 363-374.

[114] Widom, H., On the limiting distribution for the longest alternating sequence in a random permutation. Preprint; math.CO/0511533.

[115] Wilf, H., The patterns of permutations. Discrete Math. 257 (2002), 575-583.

Richard Stanley, Massachusetts Institute of Technology, Department of Mathematics, 77 Massachessetts Avenue, Cambridge, MA 02139-4307, U.S.A.

E-mail: rstan@math.mit.edu 